\title{
Generalized thermodynamics and Fokker-Planck equations. Applications to stellar dynamics and two-dimensional turbulence
}

\author{
Pierre-Henri Chavanis \\ Laboratoire de Physique Théorique, Université Paul Sabatier \\ 118 route de Narbonne, 31062 Toulouse Cedex 4, France \\ (chavanis@irsamc2.ups-tlse.fr)
}

\begin{abstract}
We introduce a new set of generalized Fokker-Planck equations that conserve energy and mass and increase a generalized entropy functional until a maximum entropy state is reached. Nonlinear Fokker-Planck equations associated with Tsallis entropies are a special case of these equations. Application of these results to stellar dynamics and vortex dynamics are proposed. Our prime result is a novel relaxation equation that should offer an easily implementable parametrization of $2 \mathrm{D}$ turbulence. Usual parametrizations (including a single turbulent viscosity) correspond to the infinite temperature limit of our model. They forget a fundamental systematic drift that acts against diffusion as in Brownian theory. Our generalized Fokker-Planck equations can have applications in other fields of physics such as chemotaxis for bacterial populations. We propose the idea of a classification of generalized entropies in "classes of equivalence" and provide an aesthetic connexion between topics (vortices, stars, bacteries,...) which were previously disconnected.
\end{abstract}

PACS numbers: 05.90.+m, 05.70.-a, 47.10.+g, 47.32.-y 


\section{INTRODUCTION}

The statistical mechanics of systems with long-range interactions is currently a topic of active research [1]. Systems with long-range interactions are numerous in nature: self-gravitating systems, two-dimensional vortices, non-neutral plasmas, metallic clusters, dipoles, fracture etc... These systems exhibit similar features such as negative specific heats, inequivalence of statistical ensembles, phase transitions and self-organization. Since they are non-extensive and non-additive, the construction of an appropriate thermodynamics is a challenging problem. Among all the previous examples, self-gravitating systems and 2D vortices play a special role because they both interact via an unshielded Newtonian potential (in dimensions $D=3$ or $D=2$ ) and possess a rather similar mathematical structure [2].

It has been recently argued that the classical Boltzmann entropy may not be correct for systems with long-range interactions and that Tsallis entropies, also called $q$-entropies, should be used instead [3]. In the context of 2D turbulence, Boghosian [4] has interpreted a result of plasma physics [5] in terms of Tsallis generalized thermodynamics. In the astrophysical context, Plastino \& Plastino [6] have noted that the maximization of Tsallis entropies leads to stellar polytropes, thereby avoiding the infinite mass problem associated with isothermal systems obtained by maximizing the Boltzmann entropy. However, the arguments advocated to justify Tsallis entropies in the context of 2D turbulence and stellar dynamics are usually unclear and misleading and were criticized in our previous papers [7-9]. In particular, we have argued that Tsallis entropies are particular $H$-functions (not true entropies) [10] whose maximization at fixed mass and energy determines (nonlinearly) dynamically stable stationary solutions of the 2D Euler or Vlasov-Poisson systems $[11,12]$. The $H$-functions can be useful in describing the metaequilibrium states resulting from incomplete violent relaxation [13]. In this context, the aforementioned maximization problem is a condition of dynamical stability, not a condition of thermodynamical stability. Therefore, Tsallis entropies have not a fundamental justification for stellar systems and 2D turbulence. They form just a one-parameter family of $H$-functions that leads to simple models (stellar polytropes and polytropic vortices). Tsallis distributions can provide, however, a convenient fit of the metaequilibrium state in case of incomplete relaxation. In that context, the parameter $q$, which can vary in space, measures the importance of mixing [9].

On the other hand, it has been shown that Tsallis generalized thermodynamics could be useful to interpret anomalous diffusion in complex systems and that the $q$-entropies are connected to nonlinear Fokker-Planck equations [14]. In fact, Tsallis entropies are just a particular class of a much larger class of functionals that we shall call generalized entropies. These functionals are defined as $S=-\int C(f) d^{3} \mathbf{r} d^{3} \mathbf{v}$ where $C(f)$ is a convex function of the distribution function. Many important properties obtained with Tsallis $q$-entropies (nonlinear Fokker-Planck equations, generalized $H$-theorem, Legendre transforms,...) remain valid for these more general functionals. Tsallis entropies give a special importance to power-laws. Power-laws are indeed important in physics (in relation, among other, with multifractality [3]) but they are not the most general distributions. Generalized entropies arise naturally when the diffusion coefficient is an arbitrary function $D(f)$ of the distribution function. When the diffusion is counter-balanced by a friction or a drift, they play the role of Lyapunov functionals and satisfy a $H$-theorem $\dot{S} \geq 0$. In this context, Tsallis entropies correspond to a power-law dependance of the diffusion coefficient $D(f) \sim f^{q-1}$ and the 
$q$-parameter in Tsallis formalism is related to the exponent of anomalous diffusion.

In the first part of the paper, we develop a generalized thermodynamical formalism for a large class of entropy functionals encompassing Boltzmann, Fermi and Tsallis entropies. This formalism can have applications in different domains of physics (or biology, economy, mathematics,...) with different interpretations. In Sec. II A, we introduce generalized entropies that extend those introduced by Tsallis and co-workers. In Sec. II B, we establish the conditions of generalized thermodynamical stablity in microcanonical and canonical ensembles and discuss the possible inequivalence of statistical ensembles when the caloric curve presents turning points or bifurcations. In Secs. II C and II D, we introduce generalized kinetic equations (Fokker-Planck, Landau) that conserve mass and energy and increase a generalized entropy functional instead of the Boltzmann entropy. In Sec. III, we study the generalized Smoluchowski-Poisson system and mention possible applications to bacterial populations (chemotaxis).

In the second part of the paper (Sec. IV), we discuss the statistical mechanics of Hamiltonian systems with long-range interactions focusing on stellar systems and 2D point vortices (or inviscid continuous vorticity fields). We consider the collisionless regime when the $N \rightarrow+\infty$ limit is taken before the $t \rightarrow+\infty$ limit (Vlasov limit). Due to mean-field effects and long-range interactions, the system undergoes a violent relaxation (Sec. IV A). The resulting metaequilibrium state is a stationary solution of the Vlasov (or Euler) equation on the coarse-grained scale. Its nonlinear dynamical stability can be settled via a thermodynamical analogy (Sec. IV B). Generalized entropies (also called H-functions) arise due to the existence of fine-grained constraints (Casimirs), non-ergodicity and non-ideal effects (forcing, dissipation,...). We propose a novel relaxation equation (Sec. IV C) that can serve either as a small-scale parametrization of 2D turbulence (Sec. IV E) or as a powerful numerical algorithm to compute arbitrary nonlinearly dynamically stable stationary solutions of the 2D Euler-Poisson system (Sec. IV D). We propose the idea of a classification of generalized entropies in "classes of equivalence" with the heuristic argument that entropies of the same class should lead to similar results (Sec. IV F).

\section{GENERALIZED THERMODYNAMICS AND FOKKER-PLANCK EQUATIONS}

\section{A. Generalized entropies}

Let us consider a system of $N$ particles in interaction and denote by $f(\mathbf{r}, \mathbf{v}, t)$ their distribution function defined such that $f d^{3} \mathbf{r} d^{3} \mathbf{v}$ gives the total mass of particles with position $\mathbf{r}$ and velocity $\mathbf{v}$ at time $t$. Let $\mathbf{F}(\mathbf{r}, t)=-\nabla \Phi$ be the force (by unit of mass) experienced by a particle. We assume that the potential $\Phi(\mathbf{r}, t)$ is related to the density $\rho(\mathbf{r}, t)=\int f d^{3} \mathbf{v}$ by a relation of the form $\Phi(\mathbf{r})=\int \rho\left(\mathbf{r}^{\prime}\right) u\left(\mathbf{r}-\mathbf{r}^{\prime}\right) d^{3} \mathbf{r}^{\prime}$ where $u\left(\mathbf{r}-\mathbf{r}^{\prime}\right)$ is an arbitrary binary potential. For example, if $u\left(\mathbf{r}-\mathbf{r}^{\prime}\right)=-G /\left|\mathbf{r}-\mathbf{r}^{\prime}\right|, \Phi$ is solution of the Poisson equation

$$
\Delta \Phi=4 \pi G \rho .
$$

We assume that the system is isolated so that it conserves mass

$$
M=\int \rho d^{3} \mathbf{r}
$$


and energy

$$
E=\int \frac{1}{2} f v^{2} d^{3} \mathbf{r} d^{3} \mathbf{v}+\frac{1}{2} \int \rho \Phi d^{3} \mathbf{r}=K+W,
$$

where $K$ is the kinetic energy and $W$ the potential energy. The conservation of angular momentum $\mathbf{L}=\int f(\mathbf{r} \times \mathbf{v}) d^{3} \mathbf{r} d^{3} \mathbf{v}$ and linear impulse $\mathbf{P}=\int f \mathbf{v} d^{3} \mathbf{r} d^{3} \mathbf{v}$ can be easily incorporated in the formalism. The following results remain valid if $\Phi=\Phi_{\text {ext }}(\mathbf{r})$ is a fixed external potential, in which case $W=\int \rho \Phi_{e x t} d^{3} \mathbf{r}$.

We introduce a generalized entropy of the form

$$
S=-\int C(f) d^{3} \mathbf{r} d^{3} \mathbf{v}
$$

where $C(f)$ is a convex function, i.e. $C^{\prime \prime}(f)>0$. We are interested by the distribution function $f$ which maximizes the generalized entropy (4) at fixed mass and energy. Introducing appropriate Lagrange multipliers and writing the variational principle in the form

$$
\delta S-\beta \delta E-\alpha \delta M=0,
$$

we find that the critical points of entropy at fixed mass and energy are given by

$$
C^{\prime}(f)=-\beta \epsilon-\alpha,
$$

where $\epsilon=\frac{v^{2}}{2}+\Phi$ is the energy of a particle by unit of mass. The Lagrange multipliers $\beta$ and $\alpha$ are the generalized inverse temperature and the generalized chemical potential. Equation (6) can be written equivalently as

$$
f=F(\beta \epsilon+\alpha),
$$

where $F(x)=\left(C^{\prime}\right)^{-1}(-x)$. From the identity

$$
f^{\prime}(\epsilon)=-\beta / C^{\prime \prime}(f),
$$

resulting from Eq. (6), $f(\epsilon)$ is a monotonically decreasing function of energy if $\beta>0$. The conservation of angular momentum can be easily included in the variational principle (5) by introducing an appropriate Lagrange multiplier $\Omega$. Equation (6) remains valid provided that $\epsilon$ is replaced by the Jacobi energy $\epsilon_{J}=\epsilon-\boldsymbol{\Omega} \cdot(\mathbf{r} \times \mathbf{v})=\frac{1}{2}(\mathbf{v}-\boldsymbol{\Omega} \times \mathbf{r})^{2}+\Phi_{\text {eff }}$ where $\Phi_{\text {eff }}=\Phi-\frac{1}{2}(\boldsymbol{\Omega} \times \mathbf{r})^{2}$ is the effective potential accounting for inertial forces.

Among all functionals of the form (4), some have been discussed in detail in the literature. The most famous functional is the Boltzmann entropy

$$
S_{B}[f]=-\int f \ln f d^{3} \mathbf{r} d^{3} \mathbf{v} .
$$

It leads to the isothermal (or Boltzmann) distribution

$$
f=A e^{-\beta \epsilon} .
$$

Closely related to the Boltzmann entropy is the Fermi-Dirac entropy 


$$
S_{F . D .}[f]=-\int\left\{\frac{f}{\eta_{0}} \ln \frac{f}{\eta_{0}}+\left(1-\frac{f}{\eta_{0}}\right) \ln \left(1-\frac{f}{\eta_{0}}\right)\right\} d^{3} \mathbf{r} d^{3} \mathbf{v}
$$

which leads to the Fermi-Dirac distribution function

$$
f=\frac{\eta_{0}}{1+\lambda e^{\beta \eta_{0} \epsilon}} .
$$

The Fermi-Dirac distribution function (12) satisfies the constraint $f \leq \eta_{0}$ which is related to Pauli's exclusion principle in quantum mechanics. The isothermal distribution function (10) is recovered in the non-degenerate limit $f \ll \eta_{0}$. We can also consider the case of bosons with the sign + in Eq. (11). Recently, there was a considerable interest for functionals of the form

$$
S_{q}[f]=-\frac{1}{q-1} \int\left(f^{q}-f\right) d^{3} \mathbf{r} d^{3} \mathbf{v},
$$

where $q$ is a real number. Such entropies introduced by Tsallis [3] are called $q$-entropies. They lead to "polytropic" distributions of the form

$$
f=A(\lambda-\epsilon)^{n-\frac{3}{2}},
$$

with $A=[(q-1) \beta / q]^{\frac{1}{q-1}}$ and $\lambda=[1-(q-1) \alpha] /(q-1) \beta$. The index $n$ of the polytrope is related to the parameter $q$ by the relation $n=3 / 2+1 /(q-1)$. Isothermal distribution functions are recovered in the limit $q \rightarrow 1$ (i.e. $n \rightarrow+\infty$ ).

In any system with $f=f(\epsilon)$, one may define a local energy dependant excitation temperature by the relation

$$
\frac{1}{T(\epsilon)}=-\frac{d \ln f}{d \epsilon}
$$

For the isothermal distribution (10), $T(\epsilon)$ coincides with the thermodynamic temperature $T=1 / \beta$. For the polytropic distribution $(14), T(\epsilon)=(q-1)(\lambda-\epsilon)$. This excitation temperature has a constant gradient $d T / d \epsilon=q-1$ related to Tsallis $q$-parameter (or equivalently to the index $n$ of the polytrope). The other parameter $\lambda$ is related to the value of energy where the temperature reaches zero.

\section{B. Generalized thermodynamical stability}

In the preceding section, we have just determined critical points of the generalized entropy (4) by cancelling its first order variations with appropriate constraints. We now turn to the thermodynamical stability of the solutions (in the generalized sense). We must select maxima of $S[f]$ at fixed mass and energy. The condition that $f$ is a maximum of $S$ at fixed mass and energy is equivalent to the condition that $\delta^{2} J \equiv \delta^{2} S-\beta \delta^{2} E$ is negative for all perturbations that conserve mass and energy to first order. This condition can be written

$$
\begin{gathered}
\delta^{2} J=-\int C^{\prime \prime}(f) \frac{(\delta f)^{2}}{2} d^{3} \mathbf{r} d^{3} \mathbf{v}-\frac{1}{2} \beta \int \delta \rho \delta \Phi d^{3} \mathbf{r} \leq 0, \\
\forall \delta f \mid \delta E=\delta M=0 .
\end{gathered}
$$


So far, we have implicitly worked in the microcanonical ensemble in which the energy is fixed. However, it may be of interest to study in parallel the canonical ensemble in which the temperature $T=1 / \beta$ is fixed instead of the energy. In that case, the appropriate thermodynamical potential is the free energy $F=E-T S$ that we write for convenience in the form of Massieu function

$$
J=S-\beta E .
$$

According to Eq. (5), we have

$$
\delta J=-E \delta \beta+\alpha \delta M
$$

Therefore, the equilibrium state in the canonical ensemble is a maximum of $J$ at fixed mass and temperature. If we just cancel the first order variations of $J$, this again yields the relation (6). The condition of thermodynamical stability in the canonical ensemble requires that $f$ is a maximum of $J$ at fixed mass and temperature. This is equivalent to the condition that $\delta^{2} J$ is negative for all perturbations that conserve mass. This can be written

$$
\begin{gathered}
\delta^{2} J=-\int C^{\prime \prime}(f) \frac{(\delta f)^{2}}{2} d^{3} \mathbf{r} d^{3} \mathbf{v}-\frac{1}{2} \beta \int \delta \rho \delta \Phi d^{3} \mathbf{r} \leq 0, \\
\forall \delta f \mid \delta M=0 .
\end{gathered}
$$

We note that canonical stability implies microcanonical stability but the converse is wrong in general. Indeed, if inequality (19) is satisfied for all perturbations that conserve mass, it is a fortiori satisfied for perturbations that conserve mass and energy. Since the converse is wrong, this implies that we can "miss" some relevant solutions by working in the canonical ensemble instead of the microcanonical one.

For self-gravitating systems described by the Boltzmann entropy (9), it is well-known that the statistical ensembles are non-equivalent $[15,2,9]$. Indeed, an isothermal distribution (10) can be stable in the microcanonical ensemble (maximum of $S_{B}$ at fixed $M$ and $E$ ) but unstable in the canonical ensemble (minimum or saddle point of $J_{B}$ at fixed $M$ and $T$ ). In fact, the inequivalence of statistical ensembles for systems with long-range interactions is not limited to self-gravitating systems nor to the Boltzmann entropy (9). It occurs for many other physical systems and for various functionals of the form (4). There will be inequivalence of statistical ensembles when the caloric curve $\beta(E)$ presents turning points leading to regions of negative specific heats, or said differently, when the entropy $S(E)$ has a convex dip [16,17]. The stability of the solutions can be decided by using the turning point criterion of Katz [18] which extends the theory of Poincaré on linear series of equilibria. It is found that a change of stability in the series of equilibria occurs in the microcanonical ensemble when the energy is extremum and in the canonical ensemble when the temperature is extremum. Stability is lost or gained depending on whether the series of equilibria turns clockwise or anti-clockwise at that critical point. A change of stability along a series of equilibria can also occur at a branching point $[18,19]$, where the solutions bifurcate. A general classification of phase transitions for systems with long-range interactions has been proposed recently by Bouchet \& Barré [20]. 


\section{Generalized Kramers equation}

We shall now introduce a generalized Fokker-Planck equation, consistent with the thermodynamical framework developed previously, by using a Maximum Entropy Production Principle [21]. To apply the MEPP, we first write the relaxation equation for the distribution function in the form

$$
\frac{\partial f}{\partial t}+\mathbf{U}_{6} \cdot \nabla_{6} f=-\frac{\partial \mathbf{J}_{f}}{\partial \mathbf{v}}
$$

where $\mathbf{U}_{6}=(\mathbf{v}, \mathbf{F})$ is a generalized velocity field in the six-dimensional phase space $\{\mathbf{r}, \mathbf{v}\}$, $\nabla_{6}=(\partial / \partial \mathbf{r}, \partial / \partial \mathbf{v})$ is a generalized gradient and $\mathbf{J}_{f}$ is the diffusion current to be determined. The form of Eq. (20) ensures the conservation of mass provided that $\mathbf{J}_{f}$ decreases sufficiently rapidly for large $|\mathbf{v}|$. From Eqs. (3), (4) and (20), it is easy to put the time variations of energy and entropy in the form

$$
\begin{gathered}
\dot{E}=\int \mathbf{J}_{f} \cdot \mathbf{v} d^{3} \mathbf{r} d^{3} \mathbf{v} \\
\dot{S}=-\int C^{\prime \prime}(f) \mathbf{J}_{f} \cdot \frac{\partial f}{\partial \mathbf{v}} d^{3} \mathbf{r} d^{3} \mathbf{v},
\end{gathered}
$$

where we have used straightforward integrations by parts. Following the MEPP, we shall now determine the optimal current $\mathbf{J}_{f}$ which maximizes the rate of entropy production (22) while satisfying the conservation of energy $\dot{E}=0$. For this problem to have a solution, we shall also impose a limitation on the current $\left|\mathbf{J}_{f}\right|$, characterized by a bound $C(\mathbf{r}, \mathbf{v}, t)$ which exists but is not known, so that

$$
\frac{J_{f}^{2}}{2 f} \leq C(\mathbf{r}, \mathbf{v}, t)
$$

It can be shown by a convexity argument that reaching the bound (23) is always favorable

for increasing $\dot{S}$, so that this constraint can be replaced by an equality. The variational problem can then be solved by introducing at each time $t$ Lagrange multipliers $\beta$ and $1 / D$ for the two constraints. The condition

$$
\delta \dot{S}-\beta(t) \delta \dot{E}-\int \frac{1}{D} \delta\left(\frac{J_{f}^{2}}{2 f}\right) d^{3} \mathbf{r} d^{3} \mathbf{v}=0,
$$

yields an optimal current of the form

$$
\mathbf{J}_{f}=-D\left[f C^{\prime \prime}(f) \frac{\partial f}{\partial \mathbf{v}}+\beta(t) f \mathbf{v}\right] .
$$

The time evolution of the Lagrange multiplier $\beta(t)$ is determined by the conservation of energy $\dot{E}=0$, introducing Eq. (25) in the constraint (21). This yields

$$
\beta(t)=-\frac{\int D f C^{\prime \prime}(f) \frac{\partial f}{\partial \mathbf{v}} \cdot \mathbf{v} d^{3} \mathbf{r} d^{3} \mathbf{v}}{\int D f v^{2} d^{3} \mathbf{r} d^{3} \mathbf{v}} .
$$


Note that the optimal current (25) can be written $\mathbf{J}_{f}=D f \partial \alpha / \partial \mathbf{v}$ where

$$
\alpha(\mathbf{r}, \mathbf{v}, t) \equiv-C^{\prime}(f)-\beta \epsilon,
$$

is a generalized potential which is uniform at equilibrium according to Eq. (6). Therefore, the MEPP is just a variational formulation of the linear thermodynamics of Onsager.

Introducing the optimal current (25) in Eq. (20), we obtain the generalized FokkerPlanck equation

$$
\frac{\partial f}{\partial t}+\mathbf{U}_{6} \cdot \nabla_{6} f=\frac{\partial}{\partial \mathbf{v}}\left\{D\left[f C^{\prime \prime}(f) \frac{\partial f}{\partial \mathbf{v}}+\beta(t) f \mathbf{v}\right]\right\} .
$$

Morphologically, Eq. (28) extends the usual Kramers equation introduced in the context of colloidal suspensions [22] and collisional stellar dynamics [23]. The first term is a generalized diffusion (depending on the distribution function) and the second term is a friction. The function $\beta(t)$ can be considered as a time dependant inverse temperature evolving with time so as to conserve energy (microcanonical formulation). The friction coefficient $\xi=D \beta$ satisfies a generalized Einstein relation. Note that $D$ is not determined by the MEPP since it is related to the unknown bound $C(\mathbf{r}, \mathbf{v}, t)$ in Eq. (23). We can use this indetermination to write Eq. (28) in the alternative form

$$
\frac{\partial f}{\partial t}+\mathbf{U}_{6} \cdot \nabla_{6} f=\frac{\partial}{\partial \mathbf{v}}\left\{D^{\prime}\left[\frac{\partial f}{\partial \mathbf{v}}+\frac{\beta(t)}{C^{\prime \prime}(f)} \mathbf{v}\right]\right\},
$$

which will have the same general properties as Eq. (28). This equation involves an ordinary diffusion and a nonlinear friction. Equation (29) can be deduced from Eq. (28) by the substitution $D^{\prime}=D f C^{\prime \prime}(f)$. One of these two forms will be prefered depending on the situation contemplated. Note that $D$ or $D^{\prime}$ can depend on $\mathbf{r}, \mathbf{v}, t$ without altering the general properties of the equations.

It is straightforward to check that Eq. (28) with the constraint (26) satisfies a $H$-theorem for the generalized entropy (4). From Eqs. (22) and (25), we can write

$$
\dot{S}=-\int \frac{\mathbf{J}_{f}}{f} \cdot\left[f C^{\prime \prime}(f) \frac{\partial f}{\partial \mathbf{v}}+\beta(t) f \mathbf{v}\right] d^{3} \mathbf{r} d^{3} \mathbf{v}+\beta(t) \int \mathbf{J}_{f} \cdot \mathbf{v} d^{3} \mathbf{r} d^{3} \mathbf{v} .
$$

The last quantity vanishes due to the conservation of energy (21). Therefore,

$$
\dot{S}=\int \frac{J_{f}^{2}}{D f} d^{3} \mathbf{r} d^{3} \mathbf{v}
$$

which is positive provided that $D>0$. Now, at equilibrium $\dot{S}=0$, hence $\mathbf{J}_{f}=\mathbf{0}$, so that according to Eq. (25),

$$
\frac{\partial C^{\prime}(f)}{\partial \mathbf{v}}+\beta \mathbf{v}=\mathbf{0}
$$

Integrating with respect to $\mathbf{v}$, we get

$$
C^{\prime}(f)=-\beta \frac{v^{2}}{2}+A(\mathbf{r}) .
$$


The cancellation of the advective term $\mathbf{U}_{6} \cdot \nabla_{6}$ in Eq. (28) combined with Eq. (33) implies that $f=f(\epsilon)$ and $\nabla A=-\beta \nabla \Phi$. Therefore, $A(\mathbf{r})=-\beta \Phi(\mathbf{r})-\alpha$ and we recover Eq. (6) with $\beta=\lim _{t \rightarrow+\infty} \beta(t)$. Therefore, a stationary solution of Eq. (28) extremizes the entropy at fixed energy and mass. In addition, only maxima of $S$ at fixed $M$ and $E$ are linearly stable with respect to the generalized Fokker-Planck equation (28). Indeed, considering the linear stability of a stationary solution of Eqs. (28) and (26), we can derive the general relation (see Appendix A)

$$
2 \lambda \delta^{2} J=\delta^{2} \dot{S} \geq 0
$$

connecting the growth rate $\lambda$ of the perturbation $\delta f \sim e^{\lambda t}$ to the second order variations of the free energy $J=S-\beta E$ and the second order variations of the rate of entropy production $\delta^{2} \dot{S} \geq 0$. Since the product $\lambda \delta^{2} J$ is positive, we conclude that a stationary solution of the generalized Fokker-Planck equation (28) is linearly stable $(\lambda<0)$ if and only if it is an entropy maximum at fixed mass and energy (see Sec. II B). This aesthetic formula shows the equivalence between dynamical and thermodynamical stability for our generalized Fokker-Planck equations. Therefore, they only select maxima of $S$, not minima or saddle points.

A relaxation equation appropriate to the canonical situation can be obtained by maximizing $\dot{J}=\dot{S}-\beta \dot{E}$ with the constraint (23). The variational principle

$$
\delta \dot{J}-\int \frac{1}{D} \delta\left(\frac{J_{f}^{2}}{2 f}\right) d^{3} \mathbf{r} d^{3} \mathbf{v}=0
$$

again yields an optimal current of the form (25) but with constant $\beta$. Since

$$
\dot{J}=-\int \mathbf{J}_{f} \cdot\left[C^{\prime \prime}(f) \frac{\partial f}{\partial \mathbf{v}}+\beta \mathbf{v}\right] d^{3} \mathbf{r} d^{3} \mathbf{v}=\int \frac{J_{f}^{2}}{D f} d^{3} \mathbf{r} d^{3} \mathbf{v} \geq 0
$$

according to Eqs. (21), (22) and (25), we find that the free energy $J$ increases monotonically until an equilibrium state of the form (6) is reached. In the canonical ensemble, we can show that $2 \lambda \delta^{2} J=\delta^{2} \dot{J} \geq 0$ and conclude that a stationary solution of the generalized FokkerPlanck equation (28) with constant $\beta$ is linearly stable if and only if it is a maximum of free energy at fixed mass and temperature (see Sec. II B).

We can also use the MEPP to construct a more general relaxation equation. Assuming that the diffusion current in Eq. (20) depends on $\mathbf{v}$ and $\mathbf{r}$ and repeating the same steps as before, we get

$$
\frac{\partial f}{\partial t}+\mathbf{U}_{6} \cdot \nabla_{6} f=\nabla_{6}\left\{D\left[f C^{\prime \prime}(f) \nabla_{6} f+\beta(t) f \mathbf{U}_{6 \perp}\right]\right\}
$$

with

$$
\beta(t)=-\frac{\int D f C^{\prime \prime}(f) \nabla_{6} f \mathbf{U}_{6 \perp} d^{3} \mathbf{r} d^{3} \mathbf{v}}{\int D f\left(\mathbf{U}_{6 \perp}\right)^{2} d^{3} \mathbf{r} d^{3} \mathbf{v}},
$$

where $\mathbf{U}_{6 \perp}=(-\mathbf{F}, \mathbf{v})$. The generalized velocity $\mathbf{U}_{6}$ in phase space is very similar to the velocity field of a two-dimensional incompressible fluid (see Sec. IV A). We note in particular that $\mathbf{U}_{6 \perp}=\nabla_{6} \epsilon$, where $\epsilon=\frac{v^{2}}{2}+\Phi$ plays the role of a generalized streamfunction. 
To conclude this section, it can be of interest to discuss some special cases explicitly. For the Boltzmann entropy $(9), C^{\prime \prime}(f)=1 / f$ and Eq. (28) has the form of an ordinary Kramers equation

$$
\frac{\partial f}{\partial t}+\mathbf{U}_{6} \cdot \nabla_{6} f=\frac{\partial}{\partial \mathbf{v}}\left[D\left(\frac{\partial f}{\partial \mathbf{v}}+\beta f \mathbf{v}\right)\right]
$$

For the Fermi-Dirac entropy $(11), C^{\prime \prime}(f)=1 / f\left(\eta_{0}-f\right)$. In order to avoid the divergence of the term $f C^{\prime \prime}(f)$ as $f \rightarrow \eta_{0}$, it is appropriate to consider the alternative form (29) of the generalized Kramers equation. This yields

$$
\frac{\partial f}{\partial t}+\mathbf{U}_{6} \cdot \nabla_{6} f=\frac{\partial}{\partial \mathbf{v}}\left\{D^{\prime}\left[\frac{\partial f}{\partial \mathbf{v}}+\beta f\left(\eta_{0}-f\right) \mathbf{v}\right]\right\}
$$

which has been initially proposed in [21]. Finally, for the Tsallis entropy $(13), C^{\prime \prime}(f)=q f^{q-2}$ and Eq. (28) has the form of a nonlinear Fokker-Planck equation

$$
\frac{\partial f}{\partial t}+\mathbf{U}_{6} \cdot \nabla_{6} f=\frac{\partial}{\partial \mathbf{v}}\left[D\left(\frac{\partial f^{q}}{\partial \mathbf{v}}+\beta f \mathbf{v}\right)\right]
$$

This equation has been studied in detail recently in relation with Tsallis entropy and anomalous diffusion [14]. The underlying mechanism giving rise to anomalous diffusion may differ depending on the physical system: Lévy walkers, porous media, vortex dipoles in 2D turbulence, etc... In such systems, the diffusion coefficient $\sim f^{q-1}$ is a power-law of the distribution function and the phase space has a fractal or multifractal structure (the exponent $q$ is related to the fractal dimension). In fact, the nice properties of Eq. (41), in particular the H-theorem, go beyond the form of entropy considered by Tsallis and remain valid for all convex function $C(f)$ even if the results are not always explicit. In the context of anomalous diffusion, Eq. (28) can be obtained from a Langevin equation of the form

$$
\frac{d \mathbf{v}}{d t}=\mathbf{F}-\xi \mathbf{v}+\sqrt{2 D f\left[\frac{C(f)}{f}\right]^{\prime}} \mathbf{R}(t),
$$

where $\mathbf{R}(t)$ is a white noise. Since the function in front of $\mathbf{R}(t)$ depends on $(\mathbf{r}, \mathbf{v})$, the last term in Eq. (42) is a multiplicative noise. When $C(f)$ is a power law, Eq. (42) reduces to the stochastic equations studied by Borland [24].

\section{Generalized Landau equation}

We shall now introduce another relaxation equation tending to a state of maximum generalized entropy at fixed mass and energy. This is the generalized Landau equation

$$
\begin{gathered}
\frac{\partial f}{\partial t}+\mathbf{U}_{6} \cdot \nabla_{6} f=\frac{\partial}{\partial v^{\mu}} \int d^{3} \mathbf{v}^{\prime} K^{\mu \nu} f f^{\prime}\left[C^{\prime \prime}(f) \frac{\partial f}{\partial v^{\nu}}-C^{\prime \prime}\left(f^{\prime}\right) \frac{\partial f^{\prime}}{\partial v^{\prime} \nu}\right], \\
K^{\mu \nu}=\frac{A}{u}\left(\delta^{\mu \nu}-\frac{u^{\mu} u^{\nu}}{u^{2}}\right),
\end{gathered}
$$


where $f=f(\mathbf{r}, \mathbf{v}, t), f^{\prime}=f\left(\mathbf{r}, \mathbf{v}^{\prime}, t\right), \mathbf{u}=\mathbf{v}^{\prime}-\mathbf{v}, A$ is a constant and $C(f)$ in any convex function. We shall also consider the alternative form

$$
\frac{\partial f}{\partial t}+\mathbf{U}_{6} \cdot \nabla_{6} f=\frac{\partial}{\partial v^{\mu}} \int d^{3} \mathbf{v}^{\prime} K^{\mu \nu}\left[\frac{1}{C^{\prime \prime}\left(f^{\prime}\right)} \frac{\partial f}{\partial v^{\nu}}-\frac{1}{C^{\prime \prime}(f)} \frac{\partial f^{\prime}}{\partial v^{\prime \nu}}\right] .
$$

Morphologically, these equations extend the usual Landau equation introduced in plasma physics and collisional stellar dynamics $[25,26]$. The generalized Landau equation satisfies the conservation of mass, energy, angular momentum and linear impulse and increases a generalized entropy (H-theorem). In addition, formula (34) remains valid so that a stationary solution of the generalized Landau equation is linearly stable if and only if it is a maximum of the generalized entropy (4).

Let us consider special cases explicitly. For the Boltzmann entropy, Eq. (43) reduces to the usual Landau equation

$$
\frac{\partial f}{\partial t}+\mathbf{U}_{6} \cdot \nabla_{6} f=\frac{\partial}{\partial v^{\mu}} \int d^{3} \mathbf{v}^{\prime} K^{\mu \nu}\left(f^{\prime} \frac{\partial f}{\partial v^{\nu}}-f \frac{\partial f^{\prime}}{\partial v^{\prime \nu}}\right)
$$

For the Fermi-Dirac entropy, Eq. (45) takes the form

$$
\frac{\partial f}{\partial t}+\mathbf{U}_{6} \cdot \nabla_{6} f=\frac{\partial}{\partial v^{\mu}} \int d^{3} \mathbf{v}^{\prime} K^{\mu \nu}\left[f^{\prime}\left(\eta_{0}-f^{\prime}\right) \frac{\partial f}{\partial v^{\nu}}-f\left(\eta_{0}-f\right) \frac{\partial f^{\prime}}{\partial v^{\prime \nu}}\right] .
$$

Finally, for the Tsallis entropy, we get

$$
\frac{\partial f}{\partial t}+\mathbf{U}_{6} \cdot \nabla_{6} f=\frac{\partial}{\partial v^{\mu}} \int d^{3} \mathbf{v}^{\prime} K^{\mu \nu}\left(f^{\prime} \frac{\partial f^{q}}{\partial v^{\nu}}-f \frac{\partial f^{\prime} q}{\partial v^{\prime} \nu}\right) .
$$

This could be called the $q$-Landau equation. Contrary to the nonlinear Kramers equation (41), it seems that Eq. (48) has never been introduced previously. We shall study its properties more specifically in a future work [27]. A connexion between the (generalized) Landau equation and the (generalized) Kramers equation can be found in a thermal bath approximation. In this context, $f=f(\mathbf{v}, t)$ describes a test particle and $f^{\prime}=f\left(\mathbf{v}^{\prime}, t\right)$ describes the field particles. If we replace $f^{\prime}$ in Eq. (43) by its equilibrium value (6), then

Eq. (43) becomes equivalent to the generalized Kramers equation (28) and the diffusion coefficient can be calculated [27].

\section{THE GENERALIZED SMOLUCHOWSKI-POISSON SYSTEM}

\section{A. The high friction limit}

The Kramers-Poisson system (39)-(1) is relatively complicated because it has to be solved in a six-dimensional phase space. However, it is well-known in Brownian theory [22] that, in the high friction limit $\xi \rightarrow+\infty$ (or equivalently for large times $t \gg \xi^{-1}$ ), the velocity distribution function becomes close to the Maxwellian distribution and the evolution of the spatial density $\rho(\mathbf{r}, t)$ is governed by the Smoluchowski equation

$$
\frac{\partial \rho}{\partial t}=\nabla\left[\frac{1}{\xi}(T \nabla \rho+\rho \nabla \Phi)\right]
$$


The Smoluchowski-Poisson system has been studied in $[28,29]$. It models the dynamics of self-gravitating Brownian particles and the chemotactic aggregation of bacterial populations.

We now proceed in deriving a generalized Smoluchowski equation by taking the high friction limit of the generalized Kramers equation. We shall assume that $\beta$ is constant (canonical situation). Integrating Eq. (28) over velocity, we get the continuity equation

$$
\frac{\partial \rho}{\partial t}+\nabla(\rho \mathbf{u})=0
$$

where $\mathbf{u}=(1 / \rho) \int f \mathbf{v} d^{3} \mathbf{v}$ is the local velocity. Multiplying Eq. (28) by $\mathbf{v}$ and integrating over velocity, we get the momentum equation

$$
\frac{\partial}{\partial t}\left(\rho u_{i}\right)+\frac{\partial}{\partial x_{j}}\left(\rho u_{i} u_{j}\right)+\frac{\partial}{\partial x_{j}} P_{i j}+\rho \frac{\partial \Phi}{\partial x_{i}}=-\int D\left[f C^{\prime \prime}(f) \frac{\partial f}{\partial v_{i}}+\beta f v_{i}\right] d^{3} \mathbf{v}
$$

where $P_{i j}=\int f w_{i} w_{j} d^{3} \mathbf{v}$ is the stress tensor and $\mathbf{w}=\mathbf{v}-\mathbf{u}$ the relative velocity. Introducing the notation $\phi(f)=\int^{f} x C^{\prime \prime}(x) d x$, the first term in the collision term can be rewritten $\partial \phi(f) / \partial \mathbf{v}$ and, since it is a gradient of a function, it vanishes by integration. We are left therefore with

$$
\frac{\partial}{\partial t}\left(\rho u_{i}\right)+\frac{\partial}{\partial x_{j}}\left(\rho u_{i} u_{j}\right)+\frac{\partial}{\partial x_{j}} P_{i j}+\rho \frac{\partial \Phi}{\partial x_{i}}=-D \beta \rho u_{i}
$$

We shall close this hierarchy of equations with the Local Thermodynamical Equilibrium (LTE) condition

$$
C^{\prime}(f)=-\beta\left(\frac{w^{2}}{2}+\lambda(\mathbf{r}, t)\right)
$$

where $\lambda(\mathbf{r}, t)$ depends only on position and time. This distribution function maximizes the local density of free energy at fixed density $\rho$ and velocity $\mathbf{u}$. The Lagrange multiplier $\lambda$ is related to the spatial density $\rho(\mathbf{r}, t)$ through the relation

$$
\rho=\int f d^{3} \mathbf{v}
$$

Furthermore, since the velocity distribution in Eq. (53) is isotropic, we have $P_{i j}=p \delta_{i j}$ where $p(\mathbf{r}, t)$ is the local pressure

$$
p=\frac{1}{3} \int f w^{2} d^{3} \mathbf{v}
$$

determined by Eq. (53). From these two relations, we find that the fluid is barotropic in the sense that $p(\mathbf{r}, t)=p[\rho(\mathbf{r}, t)]$ where the function $p[]$ is completely specified by $C(f)$. We have thus obtained what might be called the damped Euler-Jeans equations

$$
\begin{gathered}
\frac{\partial \rho}{\partial t}+\nabla(\rho \mathbf{u})=0 \\
\rho \frac{d \mathbf{u}}{d t}=-\nabla p-\rho \nabla \Phi-\xi \rho \mathbf{u},
\end{gathered}
$$


where $d / d t=\partial / \partial t+\mathbf{u} \cdot \nabla$ is the material derivative. These equations were first proposed in [21]. In the high friction limit, these equations can be simplified further since, to first order in $\xi^{-1}$, we have

$$
\rho \mathbf{u}=-\frac{1}{\xi}(\nabla p+\rho \nabla \Phi)
$$

which is obtained from Eq. (57) by neglecting the advective term. Note that the high friction limit is consistent with the Local Thermodynamical Equilibrium (53). Indeed, in the high friction limit $\xi=D \beta \rightarrow+\infty$, the term in bracket in Eq. (28) must vanish so that the distribution function satisfies (53) in good approximation with $\mathbf{u}=O\left(\xi^{-1}\right)$. Inserting the relation (58) in the continuity equation (56), we get a generalized form of the Smoluchowski equation

$$
\frac{\partial \rho}{\partial t}=\nabla\left[\frac{1}{\xi}(\nabla p+\rho \nabla \Phi)\right]
$$

initially proposed in [21]. The aesthetic form of this equation, in which the pressure $p$ replaces naturally the usual term $\rho T$ in the familiar Smoluchowski equation, suggests by itself the consistency of the generalized thermodynamical formalism. The condition of stationarity in Eq. (59) corresponds to an equilibrium between the pressure force $-\nabla p$ and the mean-field force $-\rho \nabla \Phi$. This is equivalent to Eq. (6). Indeed, using Eqs. (54), (55) and (6), one has

$$
\begin{gathered}
\rho=\frac{1}{3} \int f \frac{\partial \mathbf{v}}{\partial \mathbf{v}} d^{3} \mathbf{v}=-\frac{1}{3} \int \frac{\partial f}{\partial \mathbf{v}} \cdot \mathbf{v} d^{3} \mathbf{v}=\frac{1}{3} \beta \int \frac{v^{2}}{C^{\prime \prime}(f)} d^{3} \mathbf{v}, \\
\nabla p=\frac{1}{3} \int \frac{\partial f}{\partial \mathbf{r}} v^{2} d^{3} \mathbf{v}=-\frac{1}{3} \beta \nabla \Phi \cdot \int \frac{v^{2}}{C^{\prime \prime}(f)} d^{3} \mathbf{v},
\end{gathered}
$$

so that we obtain the condition of hydrostatic equilibrium

$$
\nabla p=-\rho \nabla \Phi
$$

Finally, we can show that the generalized Smoluchowski-Poisson system (59) satisfies a form of Virial theorem

$$
\frac{1}{2} \xi \frac{d I}{d t}=2 K+W-3 p_{b} V
$$

where $I=\int \rho r^{2} d^{3} \mathbf{r}$ is the moment of inertia and $p_{b}$ the pressure on the box (assumed uniform). The proof is the same as that given in Appendix D of Ref. [29].

To conclude this section, we can consider particular forms of the generalized Smoluchowski equation. For the Boltzmann entropy (9), Eqs. (53), (54) and (55) lead to the isothermal equation of state $p=\rho T$ and to the usual form (49) of the Smoluchowski equation. The case of the Fermi-Dirac entropy (11) has been treated in [21]. For the Tsallis entropy (13), Eqs. (53), (54) and (55) lead to the polytropic equation of state $p=K \rho^{\gamma}$ with $\gamma=1+1 / n$ and Eq. (59) becomes the nonlinear Smoluchowski equation

$$
\frac{\partial \rho}{\partial t}=\nabla\left[\frac{1}{\xi}\left(K \nabla \rho^{\gamma}+\rho \nabla \Phi\right)\right] .
$$

The nonlinear Smoluchowski-Poisson system has been studied in detail in Ref. [30]. It describes self-gravitating Langevin particles experiencing anomalous diffusion. It is likely that anomalous diffusion occurs also in biological systems so that nonlinear Smoluchowski equations can find applications in the context of chemotaxis. 


\section{B. The Lyapunov functional}

In Sec. II C, it was indicated that the Lyapunov functional associated with the generalized Kramers equation (28) with a fixed inverse temperature $\beta$ is the free energy $J=S-\beta E$ with the generalized entropy (4). Therefore, the Lyapunov functional associated with the generalized Smoluchowski equation (59) will coincide with the simplified form of $J$ obtained by using the Local Thermodynamical Equilibrium condition (53), with $\mathbf{u}=\mathbf{0}$, to express $J[f]$ as a functional of $\rho$. Using Eq. (55), the energy (3) can be written

$$
E=\frac{3}{2} \int p d^{3} \mathbf{r}+\frac{1}{2} \int \rho \Phi d^{3} \mathbf{r} .
$$

On the other hand, it is shown in [9] that when $f$ is given by Eq. (53), the entropy (4) can be rewritten as

$$
S=\frac{5}{2} \beta \int p d^{3} \mathbf{r}+\beta \int \lambda \rho d^{3} \mathbf{r} .
$$

Therefore, the free energy reads

$$
J=\beta \int p d^{3} \mathbf{r}+\beta \int \lambda \rho d^{3} \mathbf{r}-\frac{1}{2} \beta \int \rho \Phi d^{3} \mathbf{r} .
$$

It is shown furthermore in [9] that Eq. (67) can be put in the equivalent form

$$
J=-\beta \int \rho \int_{0}^{\rho} \frac{p\left(\rho^{\prime}\right)}{\rho^{\prime 2}} d \rho^{\prime} d^{3} \mathbf{r}-\frac{1}{2} \beta \int \rho \Phi d^{3} \mathbf{r}+\mathrm{C} .
$$

This is the Lyapunov functional of the generalized Smoluchowski-Poisson system. Indeed, a straightforward calculation yields

$$
\dot{J}=\beta \int \frac{1}{\xi \rho}(\nabla p+\rho \nabla \Phi)^{2} d^{3} \mathbf{r} \geq 0 .
$$

Let us consider particular cases. For the Boltzmann entropy (9), $p=\rho T$, and

$$
J=-\int \rho \ln \rho d^{3} \mathbf{r}-\frac{1}{2} \beta \int \rho \Phi d^{3} \mathbf{r} .
$$

Strictly speaking, $\int_{0}^{\rho} p\left(\rho^{\prime}\right) / \rho^{\prime 2} d \rho^{\prime}$ diverges logarithmically as $\rho^{\prime} \rightarrow 0$. This means that the general formula (68) is only marginally correct for an isothermal equation of state. However, assuming a Boltzmann distribution (10) since the begining (see [9]), we can check that Eq. (70) is indeed the right formula. Finally, for the Tsallis entropy (13), $p=K \rho^{\gamma}$ with $\gamma=1+1 / n$, so that

$$
J=-\beta n \int p d^{3} \mathbf{r}-\frac{1}{2} \beta \int \rho \Phi d^{3} \mathbf{r}+\mathrm{C}_{n},
$$

where the constant can depend on $n$. Assuming a Tsallis distribution (14) since the begining (see [9]), we find that the free energy reads 


$$
J=-\frac{1}{\gamma-1} \int\left(\beta K \rho^{\gamma}-\rho\right) d^{3} \mathbf{r}-\frac{1}{2} \beta \int \rho \Phi d^{3} \mathbf{r}
$$

This expression is consistent with Eq. (71) and it reduces to Eq. (70) in the limit $n \rightarrow+\infty$. However, it is more convenient to write the free energy in the form

$$
J=-\frac{\beta K}{\gamma-1} \int\left(\rho^{\gamma}-\rho\right) d^{3} \mathbf{r}-\frac{1}{2} \beta \int \rho \Phi d^{3} \mathbf{r},
$$

which is also consistent with Eqs. (71) and (70). Under this form, we can say that the high friction limit of a $q$-Tsallis free energy in phase space is a $\gamma$-Tsallis free energy in configuration space with $\gamma=(5 q-3) /(3 q-1)$. We note also that $-J / \beta$ can be written " $\mathcal{E}-K \mathcal{S}$ " which is similar to a free energy with $K$ playing the role of a "polytropic temperature" (see [8]). It is not clear whether this formal result bears more physical content than is apparent at first sights.

The generalized Smoluchowski equation (59) can also be written in the form

$$
\frac{\partial \rho}{\partial t}=\nabla\left[\frac{1}{\xi}\left(p^{\prime}(\rho) \nabla \rho+\rho \nabla \Phi\right)\right]
$$

If we introduce a convex function $C(\rho)$ through the relation

$$
\rho C^{\prime \prime}(\rho)=\beta p^{\prime}(\rho)
$$

we have equivalently

$$
\frac{\partial \rho}{\partial t}=\nabla\left[D\left(\rho C^{\prime \prime}(\rho) \nabla \rho+\beta \rho \nabla \Phi\right)\right]
$$

where we have defined $D=1 / \beta \xi$. Noting that Eq. (75) is equivalent to

$$
C(\rho)=\beta \rho \int_{0}^{\rho} \frac{p\left(\rho^{\prime}\right)}{\rho^{2}} d \rho^{\prime}+C_{1} \rho+C_{2}
$$

the Lyapunov functional (68) can be rewritten

$$
J=-\int C(\rho) d^{3} \mathbf{r}-\frac{1}{2} \beta \int \rho \Phi d^{3} \mathbf{r},
$$

where the term proportional to the mass has been droped. The stationary solution of the generalized Smoluchowski equation (76) is given by

$$
C^{\prime}(\rho)=-\beta \Phi-\alpha
$$

It can be obtained by maximizing the free energy (78) at fixed mass and temperature. Similarly, the generalized Smoluchowski equation (76) can be obtained by maximizing the rate of free energy production $\dot{J}$ at fixed mass and temperature (see Sec. IV C).

The case of Boltzmann and Tsallis entropies in configuration space has been discussed previously. The Fermi-Dirac entropy in configuration space $S[\rho]=-\int\left\{\rho \ln \rho+\left(\rho_{0}-\rho\right) \ln \left(\rho_{0}-\right.\right.$ $\rho)\} d^{3} \mathbf{r}$ leads to an equation of state $p(\rho)=-T \ln \left(1-\rho / \rho_{0}\right)$. For $T \rightarrow+\infty, p=\rho T$ and for 
$T \rightarrow 0, \rho$ is a step function with $\rho \sim \rho_{0}$ in the core and $\rho=0$ outside. The corresponding generalized Smoluchowski equation can be written

$$
\frac{\partial \rho}{\partial t}=\nabla\left[D^{\prime}\left(\nabla \rho+\beta \rho\left(\rho_{0}-\rho\right) \nabla \Phi\right)\right] .
$$

Coming back to the general case and expliciting the relation between $\Phi$ and $\rho$, we can rewrite Eq. (76) in the form

$$
\frac{\partial \rho}{\partial t}=\nabla\left[D\left\{\rho C^{\prime \prime}(\rho) \nabla \rho+\beta \rho \int \rho\left(\mathbf{r}^{\prime}, t\right) \frac{\partial u}{\partial \mathbf{r}}\left(\mathbf{r}-\mathbf{r}^{\prime}\right) d^{3} \mathbf{r}^{\prime}\right\}\right] .
$$

The stationary solution of this equation is determined by the integro-differential equation

$$
C^{\prime}(\rho)=-\beta \int \rho\left(\mathbf{r}^{\prime}\right) u\left(\mathbf{r}-\mathbf{r}^{\prime}\right) d^{3} \mathbf{r}^{\prime}-\alpha .
$$

Equation (81) is one of the most important result of this paper. The general study of this equation appears to be of considerable interest in view of its different potential applications. This project has been initiated by [28-30] in particular cases.

\section{Generalized stability analysis}

The equivalence between dynamical and thermodynamical stability for the generalized Smoluchowski equation (59) is proved in Appendix A. In fact, we can go further and reduce the stability problem to an eigenvalue equation as was done in the special case of isothermal and polytropic distributions $[28,30]$. Let $\rho$ and $\Phi$ refer to a stationary solution of Eq. (59) and consider a small perturbation $\delta \rho$ that conserves mass. We restrict ourselves to spherically symmetric perturbations (non spherically symmetric perturbations do not induce instability for non-rotating bodies). Writing $\delta \rho \sim e^{\lambda t}$ and expanding Eq. (59) to first order, we find that

$$
\lambda \delta \rho=\frac{1}{r^{2}} \frac{d}{d r}\left[\frac{r^{2}}{\xi}\left(\frac{d \delta p}{d r}+\delta \rho \frac{d \Phi}{d r}+\rho \frac{d \delta \Phi}{d r}\right)\right] .
$$

It is convenient to introduce the notation

$$
\delta \rho=\frac{1}{4 \pi r^{2}} \frac{d q}{d r} .
$$

Physically, $q$ represents the mass perturbation $q(r) \equiv \delta M(r)=\int_{0}^{r} 4 \pi r^{\prime 2} \delta \rho\left(r^{\prime}\right) d r^{\prime}$ within the sphere of radius $r$. It satisfies therefore the boundary conditions $q(0)=q(R)=0$. Substituting Eq. (84) in Eq. (83) and integrating, we obtain

$$
\frac{\lambda \xi}{r^{2}} q=\frac{d}{d r}\left(\frac{p^{\prime}(\rho)}{r^{2}} \frac{d q}{d r}\right)+\frac{1}{r^{2}} \frac{d q}{d r} \frac{d \Phi}{d r}+4 \pi \rho \frac{d \delta \Phi}{d r},
$$

where we have used $q(0)=0$ to eliminate the constant of integration. Using the condition of hydrostatic equilibrium $T d \rho / d r+\rho d \Phi / d r=0$ and the Gauss theorem in perturbed form $d \delta \Phi / d r=G q / r^{2}$, we can rewrite Eq. (85) as 


$$
\frac{\lambda \xi}{4 \pi \rho r^{2}} q=\frac{1}{4 \pi \rho} \frac{d}{d r}\left(\frac{p^{\prime}(\rho)}{r^{2}} \frac{d q}{d r}\right)-\frac{1}{4 \pi \rho^{2}} \frac{1}{r^{2}} \frac{d q}{d r} \frac{d p}{d r}+\frac{G q}{r^{2}}
$$

or, alternatively,

$$
\frac{d}{d r}\left(\frac{p^{\prime}(\rho)}{4 \pi \rho r^{2}} \frac{d q}{d r}\right)+\frac{G q}{r^{2}}=\frac{\lambda \xi}{4 \pi \rho r^{2}} q
$$

with $q(0)=q(R)=0$.

Let us now determine the second order variations of the generalized free energy $J$. From Eq. (68), we find that

$$
\delta^{2} J=-\beta \int \frac{p^{\prime}(\rho)}{2 \rho}(\delta \rho)^{2} d^{3} \mathbf{r}-\frac{1}{2} \beta \int \delta \rho \delta \Phi d^{3} \mathbf{r},
$$

which must be negative (with respect to mass preserving perturbations) for generalized thermodynamical stability in the canonical ensemble. Adapting a procedure similar to that followed in $[31,29,9]$, we rewrite Eq. (88) in the form

$$
\delta^{2} J=-\beta \int_{0}^{R} \frac{p^{\prime}(\rho)}{8 \pi \rho r^{2}}\left(\frac{d q}{d r}\right)^{2} d r-\frac{1}{2} \beta \int_{0}^{R} \frac{d q}{d r} \delta \Phi d r .
$$

Integrating by parts and using the boundary conditions on $q$, we get

$$
\delta^{2} J=\beta \int_{0}^{R} q \frac{d}{d r}\left(\frac{p^{\prime}(\rho)}{8 \pi \rho r^{2}} \frac{d q}{d r}\right) d r+\frac{1}{2} \beta \int_{0}^{R} q \frac{d \delta \Phi}{d r} d r .
$$

Using the Gauss theorem, we find

$$
\delta^{2} J=\beta \int_{0}^{R} q \frac{d}{d r}\left(\frac{p^{\prime}(\rho)}{8 \pi \rho r^{2}} \frac{d q}{d r}\right) d r+\frac{1}{2} \beta \int_{0}^{R} \frac{G q^{2}}{r^{2}} d r
$$

or, equivalently,

$$
\delta^{2} J=\frac{1}{2} \beta \int_{0}^{R} d r q\left[\frac{G}{r^{2}}+\frac{d}{d r}\left(\frac{p^{\prime}(\rho)}{4 \pi \rho r^{2}} \frac{d}{d r}\right)\right] q .
$$

The second order variations of free energy will be positive (implying instability) if the differential operator which occurs in the integral has positive eigenvalues. We need therefore to consider the eigenvalue problem

$$
\left[\frac{d}{d r}\left(\frac{p^{\prime}(\rho)}{4 \pi \rho r^{2}} \frac{d}{d r}\right)+\frac{G}{r^{2}}\right] q_{\lambda}(r)=\lambda q_{\lambda}(r),
$$

with $q_{\lambda}(0)=q_{\lambda}(R)=0$. If all the eigenvalues $\lambda$ are negative, then the critical point is a maximum of free energy. If at least one eigenvalue is positive, the critical point is an unstable saddle point. The point of marginal stability in the series of equilibria is determined by the condition that the largest eigenvalue is equal to zero $\lambda=0$. We thus have to solve the differential equation 


$$
\frac{d}{d r}\left(\frac{p^{\prime}(\rho)}{4 \pi \rho r^{2}} \frac{d F}{d r}\right)+\frac{G F(r)}{r^{2}}=0
$$

with $F(0)=F(R)=0$.

We note that the eigenvalue problems (87) and (93) are similar and that they coincide for marginal stability in continuity with our previous studies [28-30]. We have also found a similar eigenvalue equation by analyzing the stability of barotropic stars with respect to the Euler-Jeans equations $[31,8,9]$. These eigenvalue equations have been solved analytically (or by using simple graphical constructions) for an isothermal and a polytropic equation of state in $[31,8,9,29,30]$. It is found that the case of marginal stability $(\lambda=0)$ coincides with the point of minimum generalized temperature $1 / \beta$ as predicted by classical turning point arguments in the canonical ensemble [18]. The structure of the perturbation profile that triggers the instability (in particular the number of nodes) has also been determined in our previous papers. The present analysis shows that the structure of the mathematical problem remains the same for a general equation of state $p=p(\rho)$ even if the solutions cannot be obtained analytically.

\section{APPLICATION TO STELLAR DYNAMICS AND 2D TURBULENCE}

\section{A. Violent relaxation and metaequilibrium state}

We consider a two-dimensional incompressible and inviscid flow evolving in a plane perpendicular to the direction $\mathbf{z}$. Let $\mathbf{u}=-\mathbf{z} \times \nabla \psi$ denote the velocity field satisfying the incompressibility condition $\nabla \cdot \mathbf{u}=0$. The streamfunction $\psi$ is related to the vorticity $\omega \mathbf{z}=\nabla \times \mathbf{u}$ by the Poisson equation $\Delta \psi=-\omega$. More generally, we can consider a relation of the form $\psi(\mathbf{r})=\int g\left(\mathbf{r}-\mathbf{r}^{\prime}\right) \omega\left(\mathbf{r}^{\prime}\right) d^{2} \mathbf{r}^{\prime}$ like, e.g., in the quasi-geostrophic model [32]. We assume that the dynamics is governed by the $2 \mathrm{D}$ Euler equation

$$
\frac{\partial \omega}{\partial t}+\mathbf{u} \nabla \omega=0
$$

This equation describes the inviscid evolution of continuous vorticity flows. It also describes the mean-field evolution of $N \gg 1$ point vortices before discrete correlations have developed (Vlasov limit) [33,2]. The 2D Euler equation conserves the circulation

$$
\Gamma=\int \omega d^{2} \mathbf{r}
$$

the energy

$$
E=\frac{1}{2} \int \omega \psi d^{2} \mathbf{r}
$$

and the vorticity moments (equivalent to the Casimirs) $\Gamma_{n}=\int \omega^{n} d^{2} \mathbf{r}$. It also conserves the angular momentum $L=\int \omega r^{2} d^{2} \mathbf{r}$ in a circular domain and the impulse $P=\int \omega y d^{2} \mathbf{r}$ in a channel (or in an infinite domain).

When coupled to the Poisson equation, the 2D Euler equation develops very complex filaments as a result of a mixing process. In this sense, the fine-grained vorticity $\omega(\mathbf{r}, t)$ never 
converges towards a stationary solution. However, if we introduce a coarse-graining procedure, the coarse-grained vorticity $\bar{\omega}(\mathbf{r}, t)$ rapidly relaxes towards a metaequilibrium state. This is called violent relaxation, chaotic mixing or inviscid relaxation. This "collisionless relaxation" is common to all Hamiltonian systems with long range interactions (stellar systems, point vortices, non-neutral plasmas, HMF model,...). It physically differs from the "collisional relaxation" (or viscous decay) which takes place for much longer times (often irrelevant). This implies that the order of the limits $t \rightarrow+\infty$ and $N \rightarrow+\infty$ (or $\nu \rightarrow 0$ ) is not interchangeable [2].

There has been some attempts to describe the metaequilibrium state in terms of statistical mechanics $[13,34-36]$. In the context of 2D hydrodynamics, the statistical metaequilibrium state maximizes the mixing entropy

$$
S[\rho]=-\int \rho \ln \rho d^{2} \mathbf{r} d \sigma
$$

while conserving circulation, energy and all the Casimirs. The mixing entropy is the Boltzmann entropy for $\rho(\mathbf{r}, \sigma)$, the density probability of finding the value $\omega=\sigma$ in $\mathbf{r}$. The most probable distribution is the Gibbs state

$$
\rho(\mathbf{r}, \sigma)=\frac{1}{Z(\mathbf{r})} \chi(\sigma) e^{-(\beta \psi+\alpha) \sigma},
$$

where $\chi(\sigma) \equiv \exp \left(-\sum_{n>1} \alpha_{n} \sigma^{n}\right)$ accounts for the conservation of the fragile moments $\Gamma_{n>1}=$ $\int \rho \sigma^{n} d \sigma d^{2} \mathbf{r}$ and $\alpha, \beta$ are the usual Lagrange multipliers for $\Gamma$ and $E$ (robust integrals) [9]. The "partition function" $Z=\int \chi(\sigma) e^{-(\beta \psi+\alpha) \sigma} d \sigma$ is determined by the local normalization condition $\int \rho d \sigma=1$. The equilibrium coarse-grained vorticity $\bar{\omega} \equiv \int \rho \sigma d \sigma$ can be expressed as

$$
\bar{\omega}=-\frac{1}{\beta} \frac{\partial \ln Z}{\partial \psi}=F(\beta \psi+\alpha)=f(\psi)
$$

Since $\nabla \bar{\omega}=f^{\prime}(\psi) \nabla \psi$ and $\mathbf{u}=-\mathbf{z} \times \nabla \psi$, this is a stationary solution of the 2D Euler equation. Taking the derivative of Eq. (100), it is easy to show that [37]:

$$
\bar{\omega}^{\prime}(\psi)=-\beta \omega_{2}, \quad \omega_{2} \equiv \int \rho(\sigma-\bar{\omega})^{2} d \sigma>0,
$$

where $\omega_{2}$ is the centered local enstrophy. We note that the relation $\bar{\omega}=f(\psi)$ is always monotonic, increasing at negative temperatures and decreasing at positive temperatures. Therefore, the coarse-grained vorticity $\bar{\omega}$ extremizes a $H$-function [10]:

$$
S=-\int C(\omega) d^{2} \mathbf{r}
$$

at fixed circulation and energy, where $C(\omega)$ is a convex function, i.e. $C^{\prime \prime}(\omega)>0$. Indeed, introducing appropriate Lagrange multipliers and writing the variational principle in the form

$$
\delta S-\beta \delta E-\alpha \delta \Gamma=0
$$

we find that the critical points of entropy at fixed circulation and energy are given by 


$$
C^{\prime}(\omega)=-\beta \psi-\alpha
$$

The conservation of angular momentum and impulse can be easily included in the variational principle (103) by introducing appropriate Lagrange multipliers $\Omega$ and $U$. Equation (104) remains valid provided that $\psi$ is replaced by the relative streamfunction $\psi^{\prime}=\psi+\frac{\Omega}{2} r^{2}-U y$. Equation (104) can be written equivalently as

$$
\omega=F(\beta \psi+\alpha)
$$

where $F(x)=\left(C^{\prime}\right)^{-1}(-x)$. From the identity

$$
\omega^{\prime}(\psi)=-\beta / C^{\prime \prime}(\omega)
$$

resulting from Eq. (104), $\omega(\psi)$ is monotonically decreasing if $\beta>0$ and monotonically increasing if $\beta<0$. Therefore, for any Gibbs state of the form (100), there exists a H-function of the form (102) that the coarse-grained vorticity $\bar{\omega}$ maximizes (at fixed $\Gamma, E$ ). It can be shown furthermore that $\bar{\omega}$ maximizes this functional. We note that $C(\omega)$ is a non-universal function which depends on the initial conditions. In general, $S[\bar{\omega}]$ is not the ordinary Boltzmann entropy $S_{B}[\omega]=-\int \omega \ln \omega d^{2} \mathbf{r}$ due to fine-grained constraints (Casimirs) that modify the form of entropy that we would naively expect. In the two-levels approximation $\left\{\sigma_{0}, 0\right\}$ of the statistical theory, $S[\bar{\omega}]$ is the Fermi-Dirac entropy

$$
S_{F . D .}[\bar{\omega}]=-\int\left\{\frac{\bar{\omega}}{\sigma_{0}} \ln \frac{\bar{\omega}}{\sigma_{0}}+\left(1-\frac{\bar{\omega}}{\sigma_{0}}\right) \ln \left(1-\frac{\bar{\omega}}{\sigma_{0}}\right)\right\} d^{2} \mathbf{r}
$$

leading to the distribution

$$
\bar{\omega}=\frac{\sigma_{0}}{1+\lambda e^{\beta \sigma_{0} \psi}}
$$

In the dilute limit $\bar{\omega} \ll \sigma_{0}$, one recovers the Boltzmann entropy

$$
S_{B}[\bar{\omega}]=-\int \bar{\omega} \ln \bar{\omega} d^{2} \mathbf{r}
$$

and the isothermal vortex

$$
\bar{\omega}=A e^{-\beta \psi}
$$

Other forms of H-functions compatible with the statistical prediction (100) are presented in Sec. IV F (from now on, we drop the bar on $\omega$ except in case of ambiguity).

\section{B. Dynamical stability and thermodynamical analogy}

Unfortunately, the statistical theory of violent relaxation is not very predictive because the initial conditions are not known in practice and the Casimirs cannot be determined from the coarse-grained field once the vorticity has mixed (since $\bar{\omega}^{n} \neq \bar{\omega}^{n}$ ) [38]. In addition, the high order moments of $\omega$ are altered by non-ideal effects (viscosity, forcing,...) so that their strict conservation is abusive $[39,12]$. Finally, the relaxation is in general incomplete so that 
the ergodic hypothesis which sustains the statistical theory is not fulfilled everywhere [40,7]. One aspect of incomplete violent relaxation is that the metaequilibrium structures (vortices, galaxies,...) are more confined than predicted by statistical mechanics.

The only thing that we know for sure is that the metaequilibrium state reached by the system is a dynamically stable stationary solution of the 2D Euler-Poisson systems (on a coarse-grained scale). Ellis and collaborators [12] have shown that a strong (nonlinear) condition of dynamical stability is that $\omega$ maximizes a H-function at fixed circulation and energy. This condition of dynamical stability can be written

$$
\begin{gathered}
\delta^{2} J=-\int C^{\prime \prime}(\omega) \frac{(\delta \omega)^{2}}{2} d^{2} \mathbf{r}-\frac{1}{2} \beta \int \delta \omega \delta \psi d^{2} \mathbf{r} \leq 0, \\
\forall \delta \omega \mid \delta E=\delta \Gamma=0 .
\end{gathered}
$$

This is similar to a condition of microcanonical stability in thermodynamics. It is therefore relevant to develop a thermodynamical analogy and use the same vocabulary as in thermodynamics to analyze the dynamical stability of $2 \mathrm{D}$ flows. In this analogy, $S[\omega]$ can be called a generalized entropy, $\beta$ a generalized inverse temperature, $\ldots$ In addition, we can introduce a Legendre transform $J=S-\beta E$ which is similar to a free energy in thermodynamics. The condition that $\omega$ is a maximum of $J$ at fixed temperature and circulation can be written

$$
\begin{gathered}
\delta^{2} J=-\int C^{\prime \prime}(\omega) \frac{(\delta \omega)^{2}}{2} d^{2} \mathbf{r}-\frac{1}{2} \beta \int \delta \omega \delta \psi d^{2} \mathbf{r} \leq 0, \\
\forall \delta \omega \mid \delta \Gamma=0 .
\end{gathered}
$$

This is similar to a condition of canonical stability in thermodynamics. The criteria (111) and (112) are not equivalent if the "caloric curve" $\beta(E)$ presents turning points or bifurcations $[41,12]$. This corresponds to a situation of ensemble inequivalence in thermodynamics. Since canonical stability implies microcanonical stability (but not the converse), the stability criterion (111) is stronger than (112). This has important implications in geophysical and jovian fluid dynamics $[12,42]$.

The H-function $S[\omega]$ maximized by the system at metaequilibrium is non-universal. It depends on the initial conditions and on the strength of mixing. The Tsallis entropy

$$
S_{q}[\omega]=-\frac{1}{q-1} \int\left(\omega^{q}-\omega\right) d^{2} \mathbf{r},
$$

is a particular H-function that sometimes occurs in 2D hydrodynamics [4]. However, this is essentially fortuitous, as discussed in [7]. In addition, in this context, Tsallis functional is not a true entropy. Its maximization at fixed circulation and energy is a condition of (nonlinear) dynamical stability not a condition of thermodynamical stability. It leads to a particular class of stable stationary solutions of the 2D Euler equation characterized by

$$
\omega=A(\lambda-\psi)^{n},
$$

with $A=[(q-1) \beta / q]^{\frac{1}{q-1}}$ and $\lambda=[1-(q-1) \alpha] /(q-1) \beta$. We shall call this class of vortices polytropic vortices by analogy with stellar polytropes [26]. The index $n$ of the polytrope is related to the parameter $q$ by the relation $n=1 /(q-1)$. Isothermal vortices are recovered 
in the limit $q \rightarrow 1$ (i.e. $n \rightarrow+\infty$ ). For $q=2$, i.e. $n=1$, the relationship between $\omega$ and $\psi$ is linear [4].

To conclude this section, we note that dynamical stability results similar to (111) and (112) have been obtained for the Vlasov-Poisson system [11,9]. Therefore, the generalized thermodynamical formalism developed in Sec. II can be used to study the (nonlinear) dynamical stability of collisionless stellar systems in astrophysics and other systems with long-range interactions described by the Vlasov equation.

\section{Relaxation equations for $2 \mathrm{D}$ flows}

According to the previous discussion, an important problem in 2D hydrodynamics is to construct particular stationary solutions of the 2D Euler equation with strong stability properties. We shall consider solutions that maximize a H-function $S[\omega]$ at fixed circulation $\Gamma$ and energy $E$ [12]. The explicit construction of such solutions is non-trivial. Expoiting the thermodynamical analogy discussed previously and using the MEPP, we can obtain relaxation equations (similar to Fokker-Planck equations) that can be used as numerical algorithms to construct arbitrary stable stationary solutions of the 2D Euler equation. To apply the MEPP, we write the relaxation equation in the form

$$
\frac{\partial \omega}{\partial t}+\mathbf{u} \cdot \nabla \omega=-\nabla \cdot \mathbf{J}_{\omega}
$$

where the diffusion current $\mathbf{J}_{\omega}$ has to be determined. The form of Eq. (115) ensures the conservation of circulation provided that $\mathbf{J}_{\omega} \cdot \mathbf{n}=0$ on the domain boundary (with normal vector $\mathbf{n}$ ). From Eqs. (97), (102) and (115), it is easy to put the time variations of energy and entropy in the form

$$
\begin{gathered}
\dot{E}=\int \mathbf{J}_{\omega} \cdot \nabla \psi d^{2} \mathbf{r}, \\
\dot{S}=-\int C^{\prime \prime}(\omega) \mathbf{J}_{\omega} \cdot \nabla \omega d^{2} \mathbf{r},
\end{gathered}
$$

where we have used straightforward integrations by parts. Following the MEPP, we now determine the optimal current $\mathbf{J}_{\omega}$ which maximizes the rate of entropy production (117) while satisfying the conservation of energy $\dot{E}=0$ and the constraint

$$
J_{\omega}^{2} \leq C(\mathbf{r}, t)
$$

This maximization problem leads to the optimal current

$$
\mathbf{J}_{\omega}=-D\left[\omega C^{\prime \prime}(\omega) \nabla \omega+\beta(t) \omega \nabla \psi\right] .
$$

The time evolution of the Lagrange multiplier $\beta(t)$ is determined by introducing Eq. (119) in the energy constraint (116), using $\dot{E}=0$. This yields

$$
\beta(t)=-\frac{\int D \omega C^{\prime \prime}(\omega) \nabla \omega \cdot \nabla \psi d^{2} \mathbf{r}}{\int D \omega(\nabla \psi)^{2} d^{2} \mathbf{r}} .
$$


Introducing the optimal current (119) in Eq. (115), we obtain a relaxation equation of the form

$$
\frac{\partial \omega}{\partial t}+\mathbf{u} \cdot \nabla \omega=\nabla\left\{D\left[\omega C^{\prime \prime}(\omega) \nabla \omega+\beta(t) \omega \nabla \psi\right]\right\}
$$

The first term is a generalized diffusion and the second term is a drift. The function $\beta(t)$ can be considered as a time dependant inverse temperature (possibly negative). It evolves with time so as to conserve the total energy $E$ (microcanonical description). The drift coefficient $\xi=D \beta$ is a generalized Einstein relation. We shall use Eq. (121) when $\omega \geq 0$. When $\omega$ can be positive and negative, we shall prefer the alternative form

$$
\frac{\partial \omega}{\partial t}+\mathbf{u} \cdot \nabla \omega=\nabla\left\{D^{\prime}\left[\nabla \omega+\frac{\beta(t)}{C^{\prime \prime}(\omega)} \nabla \psi\right]\right\},
$$

Equation (122) is obtained from Eq. (121) by setting $D^{\prime}=D \omega C^{\prime \prime}(\omega)$. It is straightforward to check that Eq. (121) with the constraint (120) satisfies a $H$-theorem for the generalized entropy (102). Indeed, Eq. (117), (116) and (119) lead to

$$
\dot{S}=\int \frac{J_{\omega}^{2}}{D \omega} d^{2} \mathbf{r}
$$

where we have used $\dot{E}=0$. If $\omega \geq 0$, then Eq. (123) is positive provided that $D \geq 0$. If we use the alternative equation (122), we have $D \omega=D^{\prime} / C^{\prime \prime}(\omega)$ so that Eq. (123) is positive whatever the sign of $\omega$ provided that $D^{\prime} \geq 0$. At equilibrium $\dot{S}=0$, hence $\mathbf{J}_{\omega}=\mathbf{0}$, which is equivalent to

$$
\nabla C^{\prime}(\omega)+\beta \nabla \psi=\mathbf{0}
$$

Integrating, we get

$$
C^{\prime}(\omega)=-\beta \psi-\alpha .
$$

which returns Eq. (104) with $\beta=\lim _{t \rightarrow+\infty} \beta(t)$. Therefore, as expected, a stationary solution of Eq. (121) extremizes the generalized entropy (102) at fixed energy and circulation. In addition, it is shown in Appendix B that the relation (34) remains valid so that only maxima of $S$ at fixed $E$ and $\Gamma$ are selected by the relaxation equation. The relaxation equation appropriate to the canonical situation is obtained by maximizing $\dot{J}=\dot{S}-\beta \dot{E}$ with the constraint (118). This leads again to an optimal current of the form (119) but with constant $\beta$. In addition, Eqs. (123) and (34) remain valid with $J$ in place of $S$. Hence, the free energy $J$ increases monotonically until a state of maximum free energy is reached.

To conclude this section, we shall discuss particular cases. For the Boltzmann entropy, $C^{\prime \prime}(\omega)=1 / \omega$ and Eq. (121) reduces to

$$
\frac{\partial \omega}{\partial t}+\mathbf{u} \cdot \nabla \omega=\nabla[D(\nabla \omega+\beta \omega \nabla \psi)] .
$$

For the Fermi-Dirac entropy, $C^{\prime \prime}(\omega)=1 / \omega\left(\sigma_{0}-\omega\right)$ and Eq. (122) yields

$$
\frac{\partial \omega}{\partial t}+\mathbf{u} \cdot \nabla \omega=\nabla\left\{D^{\prime}\left[\nabla \omega+\beta \omega\left(\sigma_{0}-\omega\right) \nabla \psi\right]\right\} .
$$

For the Tsallis entropy, $C^{\prime \prime}(\omega)=q \omega^{q-2}$ and Eq. (121) becomes

$$
\frac{\partial \omega}{\partial t}+\mathbf{u} \cdot \nabla \omega=\nabla\left\{D\left[\nabla \omega^{q}+\beta \omega \nabla \psi\right]\right\} .
$$




\section{A physical numerical algorithm}

Due to the thermodynamical analogy, the relaxation equations proposed in Sec. IV C can provide a powerful numerical algorithm to compute arbitrary dynamically stable stationary solutions of the 2D Euler equation. Since we are only interested by the stationary solution, we can forget the advective term in Eq. (122) and fix $D$ to an arbitrary positive constant. We propose therefore the physical numerical algorithm

$$
\begin{gathered}
\frac{\partial \omega}{\partial t}=\nabla\left\{D\left[\nabla \omega+\frac{\beta(t)}{C^{\prime \prime}(\omega)} \nabla \psi\right]\right\}, \\
\beta(t)=-\frac{\int D \nabla \omega \nabla \psi d^{2} \mathbf{r}}{\int D \frac{(\nabla \psi)^{2}}{C^{\prime \prime}(\omega)} d^{2} \mathbf{r}} .
\end{gathered}
$$

These equations satisfy the conservation of circulation and energy (robust integrals) and increase the generalized entropy (102) until the system has reached a maximum of $S$ at

fixed $\Gamma$ and $E$. We have seen indeed that a minimum or a saddle point of $S[\omega]$ are linearly unstable via Eqs. (129)-(130). Note that specifying $C(\omega)$ does not directly determine the equilibrium state because many bifurcations can occur in parameter space $(\Gamma, E)$. There can also exist local entropy maxima (similar to metastable states in thermodynamics) leading to a complicated notion of basin of attraction (see [28] in a related context). These equations could be used to study dynamical stability problems on a new angle [12]. Indeed, if a stationary solution of Eq. (129) is stable at fixed inverse temperature $\beta$ or at fixed energy $E$, then it is nonlinearly stable with respect to the 2D Euler equation. On the other hand, if a stationary solution of Eq. (129) is stable at fixed energy $E$ (variable $\beta(t)$ ) but not at fixed inverse temperature $\beta$, then it violates Arnold's sufficient conditions of stability (see Appendix C) but is, however, dynamically stable with respect to the 2D Euler equation. The relaxation equations proposed in Sec. II could be used similarly as numerical algorithms to construct nonlinearly dynamically stable stationary solutions of the Vlasov equation.

\section{E. A simplified parametrization of $2 \mathrm{D}$ turbulence}

The generalized Fokker-Planck equations derived in Sec. IV C can also provide a simplified parametrization of 2D turbulence. The thermodynamical parametrization proposed by Robert \& Sommeria [43] can be written

$$
\frac{\partial \rho}{\partial t}+\mathbf{u} \cdot \nabla \rho=\nabla\{D[\nabla \rho+\beta(t) \rho(\sigma-\rho) \nabla \psi]\}
$$

where $\rho(\mathbf{r}, \sigma, t)$ denotes the density probability of finding the vorticity level $\sigma$ in $\mathbf{r}$ at time $t$. Equation (131) incorporates a turbulent viscosity $D$ and an additional term interpreted as a systematic drift [44]. The drift is due to the inhomogeneity of the medium and is supported by arguments of kinetic theory in simplified models (point vortices, quasilinear approximation,...) $[44,33,45]$. Usual parametrizations including a single turbulent viscosity correspond to the infinite temperature limit $(\beta=0)$ of the thermodynamical parametrization. Equation 
(131) increases the mixing entropy $S[\rho]$ while conserving the energy and all the Casimirs. For $t \rightarrow+\infty$, the solution converges to the Gibbs state (99).

The equations of Robert \& Sommeria [43] are complicated because they take into account the conservation of all the Casimirs. This clearly leads to practical difficulties. This also leads to physical difficulties because the strict conservation of all the Casimirs is abusive as discussed in IV C. We could try to simplify the problem by writing a hierarchy of equations for the moments of $\rho$. The first equation of this hierarchy is

$$
\frac{\partial \bar{\omega}}{\partial t}+\mathbf{u} \cdot \nabla \bar{\omega}=\nabla\left\{D\left[\nabla \bar{\omega}+\beta(t) \omega_{2} \nabla \psi\right]\right\},
$$

where $\omega_{2}$ is the local centered enstrophy defined in Eq. (101). However, we are now led to a difficult closure problem. Kazantsev et al. [46] have proposed to close the hierarchy of equations by a Gaussian approximation. This leads to an equilibrium state corresponding to a minimum enstrophy state. Although this state may be relevant in some particular oceanic problems (Fofonoff flows), this is not expected to be general.

In this paper, we propose to close the hierarchy of moment equations by a relation of the form

$$
\omega_{2}=\frac{1}{C^{\prime \prime}(\omega)},
$$

which can be deduced from Eqs. (101) and (106). This relation is valid at statistical equilibrium but we shall use it out of equilibrium as a convenient approximation. In a sense, this supposes that the Lagrange multipliers $\alpha_{n}$ associated to the fragile constraints $\Gamma_{n>1}$ in the Gibbs state (99) can be treated canonically [12]. Substituting Eq. (133) in Eq. (132) we obtain the simplified parametrization

$$
\begin{aligned}
\frac{\partial \omega}{\partial t}+\mathbf{u} \cdot \nabla \omega & =\nabla\left\{D\left[\nabla \omega+\frac{\beta(t)}{C^{\prime \prime}(\omega)} \nabla \psi\right]\right\}, \\
\beta(t) & =-\frac{\int D \nabla \omega \nabla \psi d^{2} \mathbf{r}}{\int D \frac{(\nabla \psi)^{2}}{C^{\prime \prime}(\omega)} d^{2} \mathbf{r}},
\end{aligned}
$$

which coincides with Eqs. (122) and (120). The function $C(\omega)$ is a non-universal function which encapsulates the complexity of the fine-grained dynamics and which depends on the situation contemplated (ocean dynamics, jovian atmosphere, decaying 2D turbulence...). For a given physical situation, we propose to select a form of $C(\omega)$ a priori and compute the corresponding equilibrium state. Then, we can check a posteriori whether it was a good choice by comparing the result with the information that we have on the system. This is similar to the notion of prior vorticity distribution proposed in [12]. Of course, this approach is essentially phenomenological and explanatory but it allows to deal with complex situations which were previously inaccessible.

To obtain an operational sub-grid scale parametrization of 2D turbulence, it remains for one to specify the value of the diffusion coefficient. Heuristic arguments $[47,21]$ or more formal kinetic theory [45] suggest that $D=K \epsilon^{2} \omega_{2}^{1 / 2}$ where $K$ is a constant of order unity and $\epsilon$ is the scale of unresolved fluctuations. This formula is a relatively direct consequence 
of the general Taylor expression of the turbulent viscosity [48] and it proved to be relevant in oceanic modelling [46]. Furthermore, it has been shown in previous works $[47,21]$ that the spatial dependance of the diffusion coefficient is important to take into account the problem of incomplete relaxation and the formation of self-confined vortices [49]. With the closure relation (133), the diffusion coefficient can be expressed in terms of $\omega$ as

$$
D=\frac{K \epsilon^{2}}{\sqrt{C^{\prime \prime}(\omega)}}
$$

The same simplifications could be introduced in the parametrization of the gravitational Vlasov-Poisson proposed in [21].

\section{F. Classification of generalized entropies}

Our parametrization involves a free function $C(\omega)$. This indetermination is intrinsic to the problem of $2 \mathrm{D}$ turbulence and not really a flaw of our approach: there is no univer-

sal entropy $S[\omega]$. In order to reduce this indetermination, we shall argue that generalized entropies can be regrouped in "classes of equivalence" with the underlying idea that functionals of the same class will produce similar results. Then, for a given physical situation, it will be possible to pick a form of $C(\omega)$ in the corresponding class of equivalence and use it in the parametrization (134)-(136).

We shall now present typical forms of "generalized entropies" $S[\omega]$ that appeared in the literature. Specifically, we shall prescribe analytical forms of the vorticity distribution $\chi(\sigma)$ in the Gibbs state (99) and compute the corresponding $C(\omega)$. These analytical expressions can be considered as prototypical examples of more realistic distributions. Consider first the case where the fine-grained vorticity takes only two values $\sigma_{0}$ and $\sigma_{1}>\sigma_{0}$ so that $\chi(\sigma)=\chi_{0} \delta\left(\sigma-\sigma_{0}\right)+\chi_{1} \delta\left(\sigma-\sigma_{1}\right)$. In that case, $\omega(\psi)$ is the distribution

$$
\omega=\sigma_{0}+\frac{\sigma_{1}-\sigma_{0}}{1+e^{\left(\sigma_{1}-\sigma_{0}\right)(\beta \psi+\alpha)}}
$$

which can also be written as a tanh,

$$
\omega=\frac{\sigma_{0}+\sigma_{1}}{2}-\frac{\sigma_{1}-\sigma_{0}}{2} \tanh \left[\frac{\sigma_{1}-\sigma_{0}}{2}(\beta \psi+\alpha)\right] .
$$

For this distribution, $\omega \rightarrow \sigma_{1}, \sigma_{0}$ when $-\beta \psi \rightarrow+\infty,-\infty$. Using Eq. (104), we get

$$
C(\omega)=p \ln p+(1-p) \ln (1-p), \quad \omega=p \sigma_{1}+(1-p) \sigma_{0}
$$

This implies

$$
C^{\prime}(\omega)=\frac{1}{\sigma_{1}-\sigma_{0}} \ln \left(\frac{\omega-\sigma_{0}}{\sigma_{1}-\omega}\right), \quad C^{\prime \prime}(\omega)=\frac{1}{\left(\omega-\sigma_{0}\right)\left(\sigma_{1}-\omega\right)} .
$$

Substituting Eq. (140) in the general parametrization (134), we obtain

$$
\frac{\partial \omega}{\partial t}+\mathbf{u} \cdot \nabla \omega=\nabla\left\{D\left[\nabla \omega+\beta(t)\left(\omega-\sigma_{0}\right)\left(\sigma_{1}-\omega\right) \nabla \psi\right]\right\}
$$


with a diffusion coefficient

$$
D=K \epsilon^{2} \sqrt{\left(\omega-\sigma_{0}\right)\left(\sigma_{1}-\omega\right)}
$$

These equations coincide with the two-levels approximation of the thermodynamical parametrization (131). This two-levels approximation has been used in relation with shear layer instability [40], vortex merging [43] and in a model of Jupiter's great red spot [50].

Turkington [38] has considered the distribution $\chi(\sigma)=\chi$ for $\sigma_{0} \leq \sigma \leq \sigma_{1}$ and $\chi(\sigma)=0$ otherwise. The choice $\alpha_{n}=0$ for $n>1$ in Eq. (99) amounts to maximizing the entropy (98) at fixed energy and circulation, neglecting the higher order vorticity moments. If we assume for simplicity that $\sigma_{0}=-\lambda$ and $\sigma_{1}=+\lambda$ then $\omega(\psi)$ is the Langevin function

$$
\omega=\lambda L[-\lambda(\beta \psi+\alpha)], \quad L(x)=\operatorname{coth}(x)-\frac{1}{x} .
$$

It does not seem possible to write down the function $C(\omega)$ explicitly. However, the relationship (143) is qualitatively similar to (138) so we argue that the Langevin-type model falls in the same "class of equivalence" as the Fermi-Dirac-type model (i.e., they will produce the same type of equilibrium states and bifurcations).

Consider now a situation in which the fine-grained vorticity can take three values $\sigma_{1}, \sigma_{2}$ and 0 so that $\chi(\sigma)=\delta(\sigma)+\chi_{1} \delta\left(\sigma-\sigma_{1}\right)+\chi_{2} \delta\left(\sigma-\sigma_{2}\right)$. Consider furthermore the dilute limit of the statistical theory in which $Z \simeq 1$. In that case, the probability of the non-zero levels is given by $p_{i}=A_{i} \exp \left[-(\beta \psi+\alpha) \sigma_{i}\right]$ and $\omega=p_{1} \sigma_{1}+p_{2} \sigma_{2}$. This dilute limit corresponds to the point vortex model [51]. If we assume furthermore, for simplicity, that $\sigma_{0}=-\lambda$ and $\sigma_{1}=+\lambda$ we get the sinh-Poisson relation

$$
\omega=-2 A \lambda \sinh (\lambda(\beta \psi+\alpha))
$$

For this distribution, $\omega \rightarrow \pm \infty$ as $-\beta \psi \rightarrow \pm \infty$. The sinh-relationship is observed in the late stages of $2 \mathrm{D}$ turbulence when the initial condition is a random vorticity field [52]. This is because the vortices that form at intermediate times are very intense and isolated as in a point vortex gas. Note that the statistical approach based on the conservation of all the Casimirs does not work in that case because of viscous effects [39]. Using Eq. (104), we find that

$$
C^{\prime}(\omega)=\frac{1}{\lambda} \sinh ^{-1}\left(\frac{\omega}{2 A \lambda}\right)
$$

Then,

$$
C(\omega)=\frac{\omega}{\lambda} \sinh ^{-1}\left(\frac{\omega}{2 A \lambda}\right)-\frac{1}{\lambda} \sqrt{4 A^{2} \lambda^{2}+\omega^{2}}
$$

and

$$
C^{\prime \prime}(\omega)=\frac{1}{\lambda} \frac{1}{\sqrt{4 A^{2} \lambda^{2}+\omega^{2}}} .
$$

Therefore, in the case of $2 \mathrm{D}$ decaying turbulence with random initial conditions, the parametrization (134) that we propose is 


$$
\frac{\partial \omega}{\partial t}+\mathbf{u} \cdot \nabla \omega=\nabla\left\{D\left[\nabla \omega+\beta(t) \lambda \sqrt{4 A^{2} \lambda^{2}+\omega^{2}} \nabla \psi\right]\right\}
$$

with a diffusion coefficient

$$
D=K \epsilon^{2} \sqrt{\lambda}\left(4 A^{2} \lambda^{2}+\omega^{2}\right)^{1 / 4}
$$

If we assumes a Poissonian weight factor $\chi(\sigma)=\exp (-|\sigma| / q)$ in Eq. (99), we get

$$
\omega=-\frac{2 q^{2}(\beta \psi+\alpha)}{1-q^{2}(\beta \psi+\alpha)^{2}} .
$$

Pasmanter [53] has noted that this relationship yields results similar to those obtained with the sinh-relationship, so they fall in the same "class of equivalence".

If we now assume that the local distribution of vorticity is Gaussian so that $\chi(\sigma)=e^{-\alpha_{2} \sigma^{2}}$ in Eq. (99), then the $\omega-\psi$ relationship is linear [35] and can be written

$$
\omega=-\Omega_{2}(\beta \psi+\alpha)
$$

where $\Omega_{2}=\omega_{2}$ is a constant equal to the centered variance of the vorticity distribution. Such a linear relationship sometimes occurs in geophysics [54]. The choice $\alpha_{n}=0$ for $n>2$ is equivalent to maximizing the entropy (98) at fixed energy, circulation and enstrophy (neglecting the higher order moments). The function $C(\omega)$ is

$$
C(\omega)=\frac{1}{2 \Omega_{2}} \omega^{2}
$$

so that the generalized entropy $S[\omega]$ is proportional to minus the (coarse-grained) enstrophy $\Gamma_{2}=\int \bar{\omega}^{2} d^{2} \mathbf{r}$. Therefore, Eq. (151) can also be obtained by minimizing the enstrophy at fixed energy and circulation [55]. A linear $\omega-\psi$ relationship is also obtained in the strong mixing limit of the statistical theory providing an inviscid justification of the minimum enstrophy principle [56].

Another choice of probability distribution has been proposed by Ellis et al. [12] in a model of jovian atmosphere where the skewness is expected to play a crucial role. They relate $\chi(\sigma)$ in the general formula (99) to the gamma density $z^{a-1} e^{-z}(z \leq 0)$ and find that

$$
\omega=\frac{-\Omega_{2}(\beta \psi+\alpha)}{1+\lambda \Omega_{2}(\beta \psi+\alpha)},
$$

where $\Omega_{2}$ is equal to the variance of $\chi(\sigma)$ and $2 \lambda \Omega_{2}^{1 / 2}$ is equal to the skewness of $\chi(\sigma)$. For this relationship, $\omega \rightarrow-\infty$ as $-(\beta \psi+\alpha) \rightarrow 1 / \lambda \Omega_{2}$ and $\omega \rightarrow-1 / \lambda$ as $-\beta \psi \rightarrow+\infty$. Therefore, the gamma model (153) is somewhat intermediate between the tanh model (138) and the sinh model (144). Using Eq. (104), we find that the corresponding generalized entropy is

$$
C(\omega)=\frac{1}{\lambda \Omega_{2}}\left[\omega-\frac{1}{\lambda} \ln (1+\lambda \omega)\right] .
$$

This yields 


$$
C^{\prime}(\omega)=\frac{\omega}{\Omega_{2}(1+\lambda \omega)}, \quad C^{\prime \prime}(\omega)=\frac{1}{\Omega_{2}(1+\lambda \omega)^{2}}
$$

For $\lambda \rightarrow 0$, we recover the gaussian model (151) as a special case of the gamma model. The parametrization (134) that we propose for this model is

$$
\frac{\partial \omega}{\partial t}+\mathbf{u} \cdot \nabla \omega=\nabla\left\{D\left[\nabla \omega+\beta(t) \Omega_{2}(1+\lambda \omega)^{2} \nabla \psi\right]\right\}
$$

with a diffusion coefficient

$$
D=K \epsilon^{2} \Omega_{2}^{1 / 2}(1+\lambda \omega)
$$

For $\lambda \rightarrow 0$ (gaussian approximation), we recover the first moment equation of the parametrization used by Kazantsev et al. [46] in a barotropic ocean model.

\section{CONCLUSION}

In this paper, we have introduced a new class of relaxation equations associated with a generalized thermodynamical framework. The Fokker-Planck and nonlinear Fokker-Planck equations corresponding to Boltzmann and Tsallis entropies are recovered as a special case. The potential applications of this new class of relaxation equations is considerable and concerns various domains of physics such as stellar dynamics, 2D turbulence, plasma physics, chemotaxis, porous media etc... For example, these equations can serve as numerical algorithms to compute arbitrary nonlinearly dynamically stable stationary solutions of VlasovPoisson or 2D Euler-Poisson systems. Physical applications of these equations and numerical simulations will be presented in future works.

We have also clarified the concept of generalized thermodynamics introduced by Tsallis [3] and applied to self-gravitating systems and two-dimensional vortices by Plastino \& Plastino [6] and Boghosian [4]. If we consider a collection of stars or point vortices and take the long time limit ( $t \rightarrow+\infty$ at fixed $N \gg 1$ ), the statistical equilibrium state resulting from a "collisional" evolution is correctly described by the ordinary Boltzmann entropy $S_{B}[f]=-\int f \ln f d^{3} \mathbf{r} d^{3} \mathbf{v}$ or $S_{B}[\omega]=-\int \omega \ln \omega d^{2} \mathbf{r}$ but the thermodynamic limit is unusual (and does not correspond to $N, V \rightarrow+\infty$ with $N / V$ fixed) [2]. In the case of stellar systems, there is no true equilibrium state but this corresponds to important physical processes (evaporation, gravothermal catastrophe) not to a breakup of thermodynamics [9]. The kinetic theory of stars and point vortices is extremely complicated due to the long-range nature of the interactions. Kinetic equations can be derived rigorously by using projection operator technics but they are non-Markovian and integro-differential $[57,33]$. The $H$-theorem for the Boltzmann entropy cannot be proved without further approximations. In addition, the diffusion coefficient depends on position, velocity and time and this can lead to a confinement of the structure in physical or phase space. These complicated effects (space and time delocalizations) can induce, for intermediate collisional times, a deviation with respect to an ideal statistical evolution. However, the infinite time limit should be described by the Boltzmann distribution.

On the other hand, for times $t \ll t_{\text {relax }}$ and $N \rightarrow+\infty$, a collection of stars or point vortices (or continuous vorticity flows) can achieve a metaequilibrium state as a result of a 
"collisionless" (or inviscid) violent relaxation. Note that the domain of validity of the collisionless regime is huge for such systems since the collisional relaxation time $t_{\text {relax }} \sim \frac{N}{\ln N} t_{D}$ is much larger than the dynamical time $t_{D}[2]$. The correct entropy is the Boltzmann entropy $S[\rho]=-\int \rho \ln \rho d \eta d^{3} \mathbf{r} d^{3} \mathbf{v}$ or $S[\rho]=-\int \rho \ln \rho d \sigma d^{2} \mathbf{r}$ for $\rho$, the local distribution of phase levels or vorticity levels. The statistical metaequilibrium state is obtained by maximizing the Boltzmann entropy $S[\rho]$ while conserving energy, circulation and an infinite class of invariants called the Casimirs. Alternatively, the coarse-grained distribution function or coarsegrained vorticity can also be obtained by maximizing a H-function $S[\bar{f}]=-\int C(\bar{f}) d^{3} \mathbf{r} d^{3} \mathbf{v}$ or $S[\bar{\omega}]=-\int C(\bar{\omega}) d^{2} \mathbf{r}$, where $C$ is a convex function, while conserving only mass (circulation) and energy (robust constraints). This functional depends on the initial conditions and is therefore non-universal. In this context, "generalized entropies" arise due to the presence of fine-grained constraints (Casimirs) that modify the macroscopic form of entropy that we would naively expect. This is a particularity of continuous Hamiltonian systems and this makes the equilibrium state difficult (if not impossible) to predict. A classification of generalized entropies in classes of equivalence can however be attempted.

Furthermore, it can happen that the mixing process during the collisionless relaxation is not sufficient to justify the ergodic hypothesis which sustains the statistical theory. In that case, the metaequilibrium state results from an incomplete violent relaxation. One possibility to take into account incomplete mixing and non-ergodicity is to introduce additional kinetic constraints in the statistical approach. This can be done by using relaxation equations with a variable diffusivity related to the local fluctuations of the vorticity or distribution function [47,21]. Accordingly, the physical picture that emerges is the following: during violent relaxation, the system has the tendency to reach the most mixed state described by the Gibbs distribution. However, as it approaches equilibrium, the mixing becomes less and less efficient and the system settles on a stationary state which is not the most mixed state. Then, the evolution is stopped until other effects (collisions, viscosity,...) come into play. The state resulting from an incomplete violent relaxation is a (nonlinearly) dynamically stable stationary solution of the Vlasov or 2D Euler equation on the coarse-grained scale. A strong condition of stability is that it maximizes a $H$-function at fixed mass (circulation) and energy $[11,12]$. Since this condition of nonlinear dynamical stability is similar to a condition of thermodynamical stability, we can use a thermodynamical analogy to study the dynamical stability of stellar systems and 2D vortices. In this analogy, the H-function can be regarded as a generalized entropy. The $\mathrm{H}$-function maximized by the system at metaequilibrium depends on the initial conditions, on the efficiency of mixing and on non-ideal effects. Tsallis entropy is just a particular $H$-function leading to polytropic distributions. It can sometimes provide a good fit of the metaequilibrium state in case of incomplete relaxation [4] but this is not general [7]. Most galaxies and 2D vortices are not described by Tsallis polytropic distribution. A better model is a composite model with an isothermal core where mixing is efficient $(q=1)$ and a polytropic halo $(q \neq 1)$ where relaxation is incomplete [9].

In conclusion, generalized entropies arise when hidden constraints are in action: Casimir invariants, kinetic constraints preventing mixing, forcing and dissipation, geometrical structure of phase space (fractality) etc... We can either work with the Boltzmann entropy and try to take into account these additional constraints or keep only the usual constraints (mass and energy) and change the form of entropy. The second possibility, while leading to some indeterminations, is often more convenient. 


\section{ACKNOWLEDGMENTS}

I thank F. Bouchet, J. Sommeria and C. Tsallis for stimulating discussions and relevant comments. I also acknowledge interesting discussions with O. Fliegans, R. Pasmanter, R. Robert, C. Rosier and B. Turkington.

\section{APPENDIX A: LINEAR STABILITY ANALYSIS OF THE GENERALIZED KRAMERS EQUATION}

Let $f$ be a stationary solution of Eq. (28) and $\delta f$ a small perturbation around this solution. Let us now linearize Eq. (28) around equilibrium and write the time dependance of the perturbation in the form $\delta f \sim e^{\lambda t}$. Noting that $\nabla_{6} \cdot \mathbf{U}_{6}=0$, we get

$$
\lambda \delta f+\nabla_{6}\left(\delta f \mathbf{U}_{6}\right)+\nabla_{6}\left(f \delta \mathbf{U}_{6}\right)=-\frac{\partial \delta \mathbf{J}_{f}}{\partial \mathbf{v}}
$$

where $\mathbf{J}_{f}$ is given by Eq. (25). Multiplying both side of Eq. (A1) by $C^{\prime \prime}(f) \delta f$ and integrating over phase space, we obtain

$$
\begin{aligned}
& \lambda \int C^{\prime \prime}(f)(\delta f)^{2} d^{3} \mathbf{r} d^{3} \mathbf{v}+\int C^{\prime \prime}(f) \delta f \nabla_{6}\left(\delta f \mathbf{U}_{6}\right) d^{3} \mathbf{r} d^{3} \mathbf{v}+ \\
& \int C^{\prime \prime}(f) \delta f \nabla_{6}\left(f \delta \mathbf{U}_{6}\right) d^{3} \mathbf{r} d^{3} \mathbf{v}=-\int C^{\prime \prime}(f) \delta f \frac{\partial \delta \mathbf{J}_{f}}{\partial \mathbf{v}} d^{3} \mathbf{r} d^{3} \mathbf{v}
\end{aligned}
$$

The second term can be rewritten

$$
I_{2}=\frac{1}{2} \int C^{\prime \prime}(f) \nabla_{6}\left[(\delta f)^{2} \mathbf{U}_{6}\right] d^{3} \mathbf{r} d^{3} \mathbf{v}=-\frac{1}{2} \int C^{\prime \prime \prime}(f)(\delta f)^{2} \nabla_{6} f \cdot \mathbf{U}_{6} d^{3} \mathbf{r} d^{3} \mathbf{v},
$$

where we have used an integration by parts. From the relation (6), we find

$$
C^{\prime \prime}(f) \nabla_{6} f=-\beta \mathbf{U}_{6 \perp},
$$

Hence, $I_{2}=0$. Using $\delta \mathbf{U}_{6}=(0,-\nabla \delta \Phi)$ and Eq. (A4), the third term in Eq. (A2) can be rewritten

$$
I_{3}=-\beta \int \delta f \mathbf{U}_{6 \perp} \delta \mathbf{U}_{6} d^{3} \mathbf{r} d^{3} \mathbf{v}=\beta \int \delta f \mathbf{v} \cdot \nabla \delta \Phi d^{3} \mathbf{r} d^{3} \mathbf{v}
$$

After an integration by parts, the fourth term in Eq. (A2) can be written

$$
I_{4}=\int \delta \mathbf{J}_{f} \cdot \frac{\partial}{\partial \mathbf{v}}\left[C^{\prime \prime}(f) \delta f\right] d^{3} \mathbf{r} d^{3} \mathbf{v}
$$

or, using Eq. (A4),

$$
I_{4}=\int \delta \mathbf{J}_{f} \cdot\left[C^{\prime \prime}(f) \frac{\partial \delta f}{\partial \mathbf{v}}-\beta \frac{C^{\prime \prime \prime}(f)}{C^{\prime \prime}(f)} \delta f \mathbf{v}\right] d^{3} \mathbf{r} d^{3} \mathbf{v} .
$$

Taking the variation of Eq. (25) and using Eq. (A4), one finds that 


$$
\delta \mathbf{J}_{f}=-D f\left[C^{\prime \prime}(f) \frac{\partial \delta f}{\partial \mathbf{v}}-\beta \frac{C^{\prime \prime \prime}(f)}{C^{\prime \prime}(f)} \delta f \mathbf{v}+\delta \beta \mathbf{v}\right]
$$

Hence,

$$
I_{4}=-\int \delta \mathbf{J}_{f} \cdot\left(\frac{\delta \mathbf{J}_{f}}{D f}+\delta \beta \mathbf{v}\right) d^{3} \mathbf{r} d^{3} \mathbf{v}=-\int \frac{\left(\delta J_{f}\right)^{2}}{D f} d^{3} \mathbf{r} d^{3} \mathbf{v} .
$$

where the last equality follows from the conservation of energy (21). Inserting the foregoing relations in Eq. (A2), we obtain

$$
\lambda \int C^{\prime \prime}(f)(\delta f)^{2} d^{3} \mathbf{r} d^{3} \mathbf{v}+\beta \int \delta f \mathbf{v} \cdot \nabla \delta \Phi d^{3} \mathbf{r} d^{3} \mathbf{v}=-\int \frac{\left(\delta J_{f}\right)^{2}}{D f} d^{3} \mathbf{r} d^{3} \mathbf{v} .
$$

We now multiply both sides of Eq. (A1) by $\delta \Phi$ and integrate over phase space. After straightforward integrations by parts, we get

$$
\lambda \int \delta f \delta \Phi d^{3} \mathbf{r} d^{3} \mathbf{v}-\int \delta f \mathbf{v} \cdot \nabla \delta \Phi d^{3} \mathbf{r} d^{3} \mathbf{v}=0 .
$$

Combining this relation with Eq. (A10), we find

$$
\lambda \int C^{\prime \prime}(f)(\delta f)^{2} d^{3} \mathbf{r} d^{3} \mathbf{v}+\lambda \beta \int \delta f \delta \Phi d^{3} \mathbf{r} d^{3} \mathbf{v}=-\int \frac{\left(\delta J_{f}\right)^{2}}{D f} d^{3} \mathbf{r} d^{3} \mathbf{v} .
$$

Now, the left hand side is just proportional to the second order variations of the free energy $\delta^{2} J$, see Eq. (16). On the other hand, recalling that $\mathbf{J}_{f}=\mathbf{0}$ at equilibrium, the second variations of the rate of entropy production (31) are given by

$$
\delta^{2} \dot{S}=\int \frac{\left(\delta J_{f}\right)^{2}}{D f} d^{3} \mathbf{r} d^{3} \mathbf{v}
$$

and they are clearly positive. Therefore, Eq. (A12) can be rewritten in the simple form $(34)$.

\section{APPENDIX B: LINEAR STABILITY ANALYSIS OF THE GENERALIZED SMOLUCHOWSKI EQUATION}

Let $\omega$ be a stationary solution of Eq. (121) and $\delta \omega$ a small perturbation around this solution. Let us now linearize Eq. (121) around equilibrium and write the time dependance of the perturbation in the form $\delta \omega \sim e^{\lambda t}$. We get

$$
\lambda \delta \omega+\nabla(\delta \omega \mathbf{u})+\nabla(\omega \delta \mathbf{u})=-\nabla \delta \mathbf{J}_{\omega},
$$

where $\mathbf{J}_{\omega}$ is given by Eq. (119). Multiplying both side of Eq. (B1) by $C^{\prime \prime}(\omega) \delta \omega$ and integrating over the whole domain, we obtain

$$
\begin{aligned}
& \lambda \int C^{\prime \prime}(\omega)(\delta \omega)^{2} d^{2} \mathbf{r}+\int C^{\prime \prime}(\omega) \delta \omega \nabla(\delta \omega \mathbf{u}) d^{2} \mathbf{r}+ \\
& \int C^{\prime \prime}(\omega) \delta \omega \nabla(\omega \delta \mathbf{u}) d^{2} \mathbf{r}=-\int C^{\prime \prime}(\omega) \delta \omega \nabla \mathbf{J}_{\omega} d^{2} \mathbf{r}
\end{aligned}
$$


The second term in Eq. (B2) can be rewritten

$$
I_{2}=\frac{1}{2} \int C^{\prime \prime}(\omega) \nabla\left[(\delta \omega)^{2} \mathbf{u}\right] d^{2} \mathbf{r}=-\frac{1}{2} \int C^{\prime \prime \prime}(\omega)(\delta \omega)^{2} \nabla \omega \cdot \mathbf{u} d^{2} \mathbf{r},
$$

where we have used an integration by parts. From the stationary condition (125), we obtain

$$
C^{\prime \prime}(\omega) \nabla \omega=-\beta \nabla \psi .
$$

Since $\mathbf{u} \cdot \nabla \psi=0$ we conclude that $I_{2}=0$. Using Eq. (B4), the third term in Eq. (B2) can be rewritten

$$
I_{3}=-\beta \int \delta \omega \delta \mathbf{u} \nabla \psi d^{2} \mathbf{r}=\beta \int \delta \omega \mathbf{u} \nabla \delta \psi d^{2} \mathbf{r} .
$$

After an integration by parts, the fourth term can be written

$$
I_{4}=\int \delta \mathbf{J}_{\omega} \cdot \nabla\left[C^{\prime \prime}(\omega) \delta \omega\right] d^{2} \mathbf{r}
$$

or, using Eq. (B4),

$$
I_{4}=\int \delta \mathbf{J}_{\omega} \cdot\left[C^{\prime \prime}(\omega) \nabla \delta \omega-\beta \frac{C^{\prime \prime \prime}(\omega)}{C^{\prime \prime}(\omega)} \delta \omega \nabla \psi\right] d^{2} \mathbf{r} .
$$

Taking the variation of Eq. (119) and using Eq. (B4), we find that

$$
\delta \mathbf{J}_{\omega}=-D \omega\left[C^{\prime \prime}(\omega) \nabla \delta \omega-\beta \frac{C^{\prime \prime \prime}(\omega)}{C^{\prime \prime}(\omega)} \delta \omega \nabla \psi+\delta \beta \nabla \psi+\beta \nabla \delta \psi\right] .
$$

Hence,

$$
I_{4}=-\int \delta \mathbf{J}_{\omega} \cdot\left(\frac{\delta \mathbf{J}_{\omega}}{D \omega}+\beta \nabla \delta \psi+\delta \beta \nabla \psi\right) d^{2} \mathbf{r}=-\int\left(\frac{\delta J_{\omega}^{2}}{D \omega}+\delta \mathbf{J}_{\omega} \beta \nabla \delta \psi\right) d^{2} \mathbf{r},
$$

where we have used the conservation of energy (116) to get the last equality. Inserting these results in Eq. (B2), we obtain

$$
\lambda \int C^{\prime \prime}(\omega)(\delta \omega)^{2} d^{2} \mathbf{r}+\beta \int \delta \omega \mathbf{u} \nabla \delta \psi d^{2} \mathbf{r}=-\int\left(\frac{\delta J_{\omega}^{2}}{D \omega}+\delta \mathbf{J}_{\omega} \beta \nabla \delta \psi\right) d^{2} \mathbf{r} .
$$

We now multiply both sides of Eq. (B1) by $\delta \psi$ and integrate over the domain. After straightforward integrations by parts, and using $\delta \mathbf{u} \cdot \nabla \delta \psi=0$, we find

$$
\lambda \int \delta \omega \delta \psi d^{2} \mathbf{r}-\int \delta \omega \mathbf{u} \cdot \nabla \delta \psi d^{2} \mathbf{r}=\int \delta \mathbf{J}_{\omega} \cdot \nabla \delta \psi d^{2} \mathbf{r} .
$$

Combining with Eq. (B10), we get

$$
\lambda \int C^{\prime \prime}(\omega)(\delta \omega)^{2} d^{2} \mathbf{r}+\lambda \beta \int \delta \omega \delta \psi d^{2} \mathbf{r}=-\int \frac{\left(\delta J_{\omega}\right)^{2}}{D \omega} d^{2} \mathbf{r} .
$$

Now, the left hand side is just proportional to the second order variations of the free energy $\delta^{2} J$, see Eq. (111). On the other hand, recalling that $\mathbf{J}_{\omega}=\mathbf{0}$ at equilibrium, the second order variations of the rate of entropy production (123) are given by

$$
\delta^{2} \dot{S}=\int \frac{\left(\delta J_{\omega}\right)^{2}}{D \omega} d^{2} \mathbf{r}
$$

and they are clearly positive (see discussion after Eq. (123)). Therefore, Eq. (B12) can be rewritten in the simple form (34). 


\section{APPENDIX C: SUFFICIENT CONDITIONS OF STABILITY AND ARNOLD THEOREMS}

According to Eqs. (106) and (111), $\delta^{2} J$ can be rewritten

$$
\delta^{2} J=-\frac{\beta}{2}\left\{\int \frac{(\delta \omega)^{2}}{-\omega^{\prime}(\psi)} d^{2} \mathbf{r}+\int \delta \omega \delta \psi d^{2} \mathbf{r}\right\} .
$$

The term is bracket is called Arnold pseudo-energy or Arnold invariant. Using an integration by parts, we have equivalently

$$
\delta^{2} J=-\frac{\beta}{2}\left\{\int \frac{(\delta \omega)^{2}}{-\omega^{\prime}(\psi)} d^{2} \mathbf{r}+\int(\nabla \delta \psi)^{2} d^{2} \mathbf{r}\right\} .
$$

First assume that $\beta>0$. By Eq. (106), we see that $\omega^{\prime}(\psi)<0$. Therefore, $\delta^{2} J<0$ and the system is stable. If $\beta>0$, then $\omega^{\prime}(\psi)>0$ and we cannot conclude directly. Let us introduce a set of normalized eigenfunctions $\phi_{i}(\mathbf{r})$ such that $-\Delta \phi_{i}=\lambda_{i} \phi_{i}$ with $\phi_{i}=0$ on the boundary of the domain and $\int \phi_{i} \phi_{j} d^{2} \mathbf{r}=\delta_{i j}$. Noting that $-\int \phi_{i} \Delta \phi_{i} d^{2} \mathbf{r}=\lambda_{i}$ and integrating by parts, we find that $\lambda_{i}=\int\left(\nabla \phi_{i}\right)^{2} d^{2} \mathbf{r}>0$. Then, we decompose $\delta \omega$ and $\delta \phi$ on these eignefunctions. Using the Poisson equation $\omega=-\Delta \psi$, we have $\delta \omega=\sum_{i} \delta \omega_{i} \phi_{i}$ and $\delta \psi=\sum_{i} \frac{\delta \omega_{i}}{\lambda_{i}} \phi_{i}$. Therefore,

$$
\int \delta \omega \delta \psi d^{2} \mathbf{r}=\sum_{i} \frac{\left(\delta \omega_{i}\right)^{2}}{\lambda_{i}}
$$

We now label the eigenvalues such that $0<\lambda_{1}<\lambda_{2}<\ldots$. Then $\lambda_{i} \geq \lambda_{1}$ and we get

$$
\int \delta \omega \delta \psi d^{2} \mathbf{r} \leq \frac{1}{\lambda_{1}} \sum_{i}\left(\delta \omega_{i}\right)^{2}=\frac{1}{\lambda_{1}} \int(\delta \omega)^{2} d^{2} \mathbf{r} .
$$

Therefore,

$$
\delta^{2} J \leq-\frac{\beta}{2} \int\left[\frac{1}{\lambda_{1}}-\frac{1}{\omega^{\prime}(\psi)}\right](\delta \omega)^{2} d^{2} \mathbf{r}
$$

As a result, if $0 \leq \omega^{\prime}(\psi)<\lambda_{1}$, then $\delta^{2} J \leq 0$ and the system is stable. Using Eq. (106), this condition can be rewritten $\beta>-\lambda_{1} \min C^{\prime \prime}(\omega)$. These sufficient conditions of stability are called Arnold theorems. They imply canonical and microcanonical stability in the thermodynamical analogy. Our general formalism shows that the Arnold theorems can have applications in many other situations than just fluid dynamics (see, e.g., [9]). 


\section{REFERENCES}

[1] T. Dauxois, S. Ruffo, E. Arimondo and M. Wilkens, in Dynamics and thermodynamics of systems with long range interactions, edited by Dauxois, T, Ruffo, S., Arimondo, E. and Wilkens, M. Lecture Notes in Physics, Springer (2002) [cond-mat/0208455].

[2] P.H. Chavanis, in Dynamics and thermodynamics of systems with long range interactions, edited by Dauxois, T, Ruffo, S., Arimondo, E. and Wilkens, M. Lecture Notes in Physics, Springer (2002) [cond-mat/0212223].

[3] C. Tsallis, J. Stat. Phys. 52, 479 (1988).

[4] B.M. Boghosian, Phys. Rev. E 53, 4754 (1996).

[5] X.P. Huang and C.F. Driscoll, Phys. Rev. Lett. 72, 2187 (1994).

[6] A. Plastino and A.R. Plastino, Phys. Lett. A 226, 257 (1997).

[7] H. Brands, P.H. Chavanis, R. Pasmanter and J. Sommeria, Phys. Fluids 11, 3465 (1999).

[8] P.H. Chavanis, Astron. Astrophys. 386, 732 (2002).

[9] P.H. Chavanis, Astron. Astrophys. 401, 15 (2003).

[10] S. Tremaine, M. Hénon and D. Lynden-Bell, Mon. Not. R. astr. Soc. 219285 (1986).

[11] J.R. Ipser and G. Horwitz, ApJ 232, 863 (1979).

[12] R. Ellis, K. Haven and B. Turkington, Nonlinearity 15, 239 (2002).

[13] D. Lynden-Bell, Mon. Not. R. astr. Soc. 136, 101 (1967).

[14] C. Tsallis and D.J. Bukman, Phys. Rev. E 54, R2197 (1996).

[15] T. Padmanabhan, Phys. Rep. 188, 285 (1990).

[16] P.H. Chavanis, Phys. Rev. E 65, 056123 (2002).

[17] P.H. Chavanis and I. Ispolatov, Phys. Rev. E 66, 036109 (2002).

[18] J. Katz, Mon. Not. R. astr. Soc. 183, 765 (1978).

[19] S. Inagaki, Progr. Th. Phys. 90, 3, 577 (1993).

[20] F. Bouchet and J. Barré, submitted to J. Stat. Phys. [cond-mat/0303307].

[21] P.H. Chavanis, J. Sommeria and R. Robert, Astrophys. J. 471, 385 (1996).

[22] H. Risken, The Fokker-Planck equation (Springer, 1989).

[23] S. Chandrasekhar, Rev. Mod. Phys. 21, 383 (1949).

[24] L. Borland, Phys. Rev. E 57, 6634 (1998).

[25] R. Balescu, Statistical Mechanics of Charged Particles (Interscience, New York, 1963).

[26] J. Binney and S. Tremaine, Galactic Dynamics, Princeton Series in Astrophysics (1987).

[27] P.H. Chavanis, submitted to Physica A [cond-mat/0304073].

[28] P.H. Chavanis, C. Rosier and C. Sire, Phys. Rev. E 66, 036105 (2002).

[29] C. Sire and P.H. Chavanis, Phys. Rev. E 66, 046133 (2002).

[30] P.H. Chavanis and C. Sire, submitted to Phys. Rev. E [cond-mat/0303088].

[31] P.H. Chavanis, Astron. Astrophys. 381, 340 (2002).

[32] J. Pedlosky, Geophysical Fluid Dynamics (Springer Verlag 1987).

[33] P.H. Chavanis, Phys. Rev. E 64, 026309 (2001).

[34] G.A. Kuzmin, in Structural Turbulence, edited by M.A. Goldshtik (Acad. Naouk CCCP Novosibirsk, Institute of ThermoPhysics, 1982).

[35] J. Miller, Phys. Rev. Lett. 65, 2137 (1990).

[36] R. Robert and J. Sommeria, J. Fluid Mech. 229, 291 (1991).

[37] P.H. Chavanis and J. Sommeria, Phys. Rev. E 65, 026302 (2002).

[38] B. Turkington, Comm. Pure. Appl. Math. 52, 781 (1999).

[39] H. Brands, J. Stulemeyer, R.A. Pasmanter and T.J. Schep, Phys. Fluids 9, 2815 (1998). 
[40] J. Sommeria, C. Staquet and R. Robert, J. Fluid Mech. 233, 661 (1991).

[41] A.R. Smith and T.M. O'Neil, Phys. Fluids B 2, 2961 (1990).

[42] F. Bouchet, PhD Thesis, Université Joseph Fourier (2001).

[43] R. Robert and J. Sommeria, Phys. Rev. Lett. 69, 2776 (1992).

[44] P.H. Chavanis, Phys. Rev. E 58, R1199 (1998).

[45] P.H. Chavanis, Phys. Rev. Lett. 84, 5512 (2000).

[46] E. Kazantzev, J. Sommeria and J. Verron, J. Phys. Ocean. 28, 1017 (1998).

[47] R. Robert and C. Rosier, J. Stat. Phys. 86, 481 (1997).

[48] G.I. Taylor, Proc. London Math. Soc. 20, 196 (1921).

[49] P.H. Chavanis and J. Sommeria, J. Fluid Mech. 356, 259 (1998).

[50] F. Bouchet and J. Sommeria, J. Fluid. Mech. 464, 165 (2002).

[51] G. Joyce and D. Montgomery, J. Plasma Phys. 10, 107 (1973).

[52] D. Montgomery, W.H. Matthaeus, W.T. Stribling, D. Martinez and S. Oughton, Phys. Fluids A 4, 3 (1992).

[53] R.A. Pasmanter, Phys. Fluids 6, 1236 (1994).

[54] N.P. Fofonoff, J. Mar. Res. 13, 254 (1954).

[55] C.E. Leith, Phys. Fluids 27, 1388 (1984).

[56] P.H. Chavanis and J. Sommeria, J. Fluid Mech. 314, 267 (1996).

[57] H.E. Kandrup, ApJ 244, 316 (1981). 